\title{
FUN3D and CFL3D Computations for the First High Lift Prediction Workshop
}

\author{
Michael A. Park* \\ Elizabeth M. Lee-Rausch* \\ Christopher L. Rumsey ${ }^{\dagger}$
}

\begin{abstract}
Two Reynolds-averaged Navier-Stokes codes were used to compute flow over the NASA Trapezoidal Wing at high lift conditions for the $1^{\text {st }}$ AIAA CFD High Lift Prediction Workshop, held in Chicago in June 2010. The unstructured-grid code FUN3D and the structured-grid code CFL3D were applied to several different grid systems. The effects of code, grid system, turbulence model, viscous term treatment, and brackets were studied. The SST model on this configuration predicted lower lift than the Spalart-Allmaras model at high angles of attack; the Spalart-Allmaras model agreed better with experiment. Neglecting viscous cross-derivative terms caused poorer prediction in the wing tip vortex region. Output-based grid adaptation was applied to the unstructured-grid solutions. The adapted grids better resolved wake structures and reduced flap flow separation, which was also observed in uniform grid refinement studies. Limitations of the adaptation method as well as areas for future improvement were identified.
\end{abstract}

\section{Introduction}

The $1^{\text {st }}$ AIAA CFD High Lift Prediction Workshop (HiLiftPW-1) was held at the $28^{\text {th }}$ Applied Aerodynamics Summer Conference in Chicago, IL during June 2010. ${ }^{a}$ HiLiftPW-1 was patterned after the successful AIAA Drag Prediction Workshops. ${ }^{1}$ The objectives of HiLiftPW-1 were to assess the numerical prediction capability of current-generation CFD for a representative three dimensional (3D) configuration. The HiLiftPW-1 test cases were based on the Trapezoidal Wing configuration, which was used in a series of NASA Langley 14- by 22-Foot Wind Tunnel and NASA Ames 12-Foot Pressurized Wind Tunnel tests.

The submissions for two NASA CFD codes are presented for simulations with several different grids as well as grid-adapted simulations based on these grids. The uniform grid refinement and adaptive grid refinement studies are primarily intended to ascertain the role of discretization error in the calculations. There are other sources of modeling error (e.g., geometry, wind tunnel corrections, boundary layer transition, and turbulence modeling) that limit the prediction of the wind tunnel measurements. Calculations with different turbulence models and brackets are also performed to better understand some of the remaining error sources.

Chaffin and Pirzadeh ${ }^{2}$ detailed the effort required to manually specify grid resolution for accurate 3D high-lift computations. Mavriplis ${ }^{3}$ showed that discretization error still dominates other error sources (e.g., turbulence model distance function calculation, thin-layer viscous approximation, level of artificial dissipation) even with extremely fine grids that are computable with today's supercomputers. To address these issues, adaptive grid methods are designed to automatically specify a resolution request that equidistributes and minimizes error estimates.

The improved resolution request is commonly based on local error estimates. ${ }^{4-7}$ Uniformly reducing the errors associated with all local-error sources of the flow may not be optimal from an engineering context, where calculating an output functional may be of greater concern. An alternative method is to estimate the error in the calculation of a specified engineering output functional. ${ }^{8-11}$ Output error indicators utilize

\footnotetext{
${ }^{*}$ Research Scientist, Computational AeroSciences Branch, NASA Langley Research Center, MS 128, Hampton, VA 23681, Senior Member AIAA.

${ }^{\dagger}$ Research Scientist, Computational AeroSciences Branch, NASA Langley Research Center, MS 128, Hampton, VA 23681, Associate Fellow AIAA.

${ }^{a}$ Data available online at http://hiliftpw.larc.nasa.gov [retrieved 11/22/2010].
} 
the dual or adjoint solution of an output functional to account for the impact of local error as well as the transport of these local errors throughout the problem domain to improve the calculation of that output functional.

The workshop summary and participant talks showed the greatest variation in the calculations for the flap and wing tip of the model. This paper is organized to begin with non-adaptive results to show trends in these sensitive flap and wing tip regions with uniform grid refinement, grid construction, geometry, and physical model. The adaptive grid results are then examined at these sensitive conditions to show common trends.

\section{CFL3D Flow Solver}

CFL3D ${ }^{12}$ is a structured-grid upwind multi-zone CFD code that solves the generalized thin-layer or full Navier-Stokes equations. It can use point-matched, patched, or overset grids and employs local time-step scaling, grid sequencing and multigrid to accelerate convergence to steady state. A time-accurate mode is available, and the code can employ low-Mach number preconditioning for accuracy in computing low-speed steady-state flows. CFL3D is a cell-centered finite-volume method. It uses third-order upwind-biased spatial differencing on the convective and pressure terms, and second-order differencing on the viscous terms; it is globally second-order accurate. Roe's flux difference-splitting method ${ }^{13}$ is used to obtain fluxes at the cell faces. The solution is advanced in time with an implicit approximate factorization method. A wide variety of turbulence models are available in the code, including linear eddy-viscosity and nonlinear models. For each loosely coupled iteration, the mean flow equations are advanced in time with the eddy-viscosity fixed then the turbulence model is advanced in time with the mean flow solution fixed. Only the one-equation model of Spalart-Allmaras ${ }^{14}$ (SA) and the two-equation shear stress transport (SST) model of Menter ${ }^{15}$ were used for this study.

\section{FUN3D Flow Solver}

FUN3D ${ }^{16-18}$ is a finite-volume Navier-Stokes solver in which the flow variables are stored at the vertices or nodes of the mesh. FUN3D solves the equations on mixed element grids, including tetrahedra, pyramids, prisms, and hexahedra and also has a two-dimensional path for triangular/quadrilateral grids. At interfaces delimiting neighboring control volumes, the inviscid fluxes are computed using an approximate Riemann solver based on the values on either side of the interface. Several convective flux schemes are available in FUN3D. The most common scheme for subsonic and transonic flows is Roe's flux difference splitting, ${ }^{13}$ which is used in the current study. For second-order accuracy, interface values are obtained by extrapolation of the control volume centroidal values, based on gradients computed at the mesh vertices using an unweighted least-squares technique. Several flux limiters are available in FUN3D to limit the reconstructed values when necessary. The most common used is that of Venkatakrishnan, ${ }^{19}$ which is used in this study. For tetrahedral meshes, the full viscous fluxes are discretized using a finite-volume formulation in which the required velocity gradients on the dual faces are computed using the Green-Gauss theorem. On tetrahedral meshes this is equivalent to a Galerkin type approximation. For non-tetrahedral meshes, the same Green-Gauss approach can lead to odd-even decoupling. A pure edge-based approach can be used to circumvent the odd-even decoupling issue, but yields only approximate viscous terms. Thus for non-tetrahedral meshes, the edgebased gradients are combined with Green-Gauss gradients, which improves the h-ellipticity of the operator, and allows the complete viscous stresses to be evaluated. ${ }^{20}$ This formulation results in a discretization of the full Navier-Stokes equations.

The solution at each time-step is updated with a backwards Euler time-differencing scheme. At each time step, the linear system of equations is approximately solved with either a multi-color point-implicit procedure or an implicit-line relaxation scheme. ${ }^{21}$ Local time-step scaling is employed to accelerate convergence to steady state. For turbulent flows, several models are available within FUN3D. In the current study, only the loosely coupled SA model ${ }^{14}$ was used for FUN3D.

\section{Output-Based Adaptation}

Output-based adaptation requires the solution of the discrete adjoint equations. ${ }^{18,22,23}$ These linear adjoint equations are solved with a dual-consistent time-marching method. ${ }^{21}$ The iterative method is con- 
structed such that an adjoint solution exactly dual to a direct differentiation approach is recovered at each time step, yielding a convergence rate which is asymptotically equivalent to that of the flow equations. To improve adjoint iterative convergence, a generalized conjugate residual (GCR) scheme ${ }^{24}$ is used with the standard multi-color point-implicit procedure as search directions.

Venditti ${ }^{10}$ described an output-based error estimation and adaptation scheme, which utilizes an embedded grid. This has been implemented in $3 \mathrm{D}^{25}$ within a parallel ${ }^{26}$ framework. The embedded grid required for the Venditti error estimator can be prohibitively large (eight times original grid) for 3D problems, which effectively limits the problem size of the output adaptive method. A single-grid error estimation procedure has been described and compared to the embedded-grid procedure for Euler problems. ${ }^{23}$ This is the first time the single-grid error estimate has been applied to turbulent simulations.

Venditti ${ }^{10}$ provided a procedure to calculate a new grid spacing request from the adaptive indicator and an user specified error tolerance. In this study, this error tolerance is set to half the total remaining error estimate at each adaptive iteration. The anisotropy of mesh elements is based on the Mach Hessian, where the element size in the smallest spacing direction is dictated by the output-based new grid spacing request. ${ }^{10}$ The anisotropic spacing request is specified by the metric $M$.

Many large-scale simulations are resource limited; it may not be practical to compute a sufficient grid size required to reach a grid converged answer. A constant-complexity scaling ${ }^{7}$ can optionally be applied to $M$ to allow the user to directly specify the size of the adapted grid. This size can be set to obtain an optimal grid that is computable with available resources. To perform this scaling, an estimate of the adapted grid size $N_{\Omega}$ is computed as

$$
N_{\Omega}=\int_{\Omega} \sqrt{\operatorname{det}(M)} d \Omega \approx \sum^{n \in \Omega} V_{n} \sqrt{\operatorname{det}\left(M_{n}\right)}
$$

where the integral is approximated by a sum over dual control volumes in the domain $\Omega$, each with a volume of $V_{n}$. The metric is scaled to produce a requested adapted grid size $N_{\text {request }}$ by

$$
\tilde{M}=\left(\frac{N_{\text {request }}}{N_{\Omega}}\right)^{\frac{2}{3}} M
$$

This specified complexity adaptation is applied to the single-grid error estimation results.

Parallel grid mechanics described by Park and Darmofal ${ }^{23,27}$ are used to modify the grid. The adaptation mechanics have only been implemented for the tetrahedral element type. The local elemental operators of node insertion, node movement, element swap, and element collapse are utilized to iteratively drive the edge lengths to unity in $M$. The grid adaptation mechanics suffer from robustness problems when performing highly anisotropic adaptation near curved boundaries. Also, the discretization in FUN3D is more accurate for semi-structured right-angle elements in the boundary layer. ${ }^{20}$ To address both of these issues, the near wall boundary layer grid is kept constant or frozen during the adaptation process. For this study, the grid is fixed within 0.14 inches of solid boundaries. This approach is not applicable to problems with initially under-resolved geometry or boundary layers, because that portion of the grid is held constant during the simulation. However, this approach is a powerful technique for problems with important off-body anisotropic features, i.e., vortices and wakes. Details of the frozen boundary layer adaptation technique are available in Andren and Park ${ }^{28}$ and Park and Carlson. ${ }^{29}$

\section{High-Lift Prediction Workshop Cases}

All of the HiLiftPW-1 workshop cases were based on a trapezoidal three-element high-lift wing (Trap wing) configuration with a simple single-slotted flap and slat. The model was developed in order to provide a database for CFD validation. This semi-span model has been tested both in the NASA Ames 12-Foot Pressurized Wind Tunnel (PWT) ${ }^{30}$ and the NASA Langley 14- by 22-Foot Subsonic Wind Tunnel (SWT). ${ }^{31}$ The SWT data was selected for the workshop. The photo of the model in the PWT, Fig. 1(a), provides a clear view of the body and brackets. The upper surface experimental pressure taps are shown in Fig. 1(b), with the locations examined in this study highlighted. Although multiple slat and flap settings were tested, the workshop test cases focused primarily on a flap landing configuration (Configuration 1). The nominal slat angle for Configuration 1 was 30 degrees, and the nominal flap angle was 25 degrees. The reference geometric quantities for the Trap wing are summarized in Table 1. Details regarding the experimental geometry, data and repeatability can be found in Slotnick et al. ${ }^{32}$ or on the HiLiftPW-1 website. While the wind tunnel 
model was tested as a semi-span model, all the workshop test cases were specified to be run in "free-air" using a computational symmetry plane at the center line of the geometry. Similarly, no wind tunnel walls or model support systems were to be included except as specified below in Case 3. The experiment did not have specified transition on the model. However in the workshop, the boundary layer was to be modeled as "fully turbulent" in the CFD simulations i.e. turbulence is modeled in all areas of computational domain. All computations were performed at a freestream Mach number of $M_{\infty}=0.2$ and freestream chord Reynolds number of $R e_{c}=4.3 \times 10^{6}$.

The first test case (Case 1) for the workshop was a grid convergence study for Configuration 1 at two angles of attack: $\alpha=13^{\circ}$ and $28^{\circ}$. A family of three grids was required for the study with consistent levels of refinement between each grid: coarse, medium, and fine. An additional extra-fine mesh solution was optional. The second test case (Case 2) for the workshop was a flap deflection study. The flap deflection study required an angle-of-attack sweep for the Trap wing with two different flap deflection angles: $25^{\circ}$ (Configuration 1) and $30^{\circ}$ (Configuration 8). For the flap deflection study, computations were performed on a set of medium-sized grids. Angle of attack computations were performed at $6^{\circ}, 13^{\circ}, 21^{\circ}, 28^{\circ}, 32^{\circ}, 34^{\circ}$ and $37^{\circ}$. The optional slat and flap support effects study (Case 3) was a comparison of Case 1 clean wing results on the medium grid size with results from a grid including the slat and flap brackets. Rumsey et al. ${ }^{33}$ provides a summary of all of the workshop submissions.

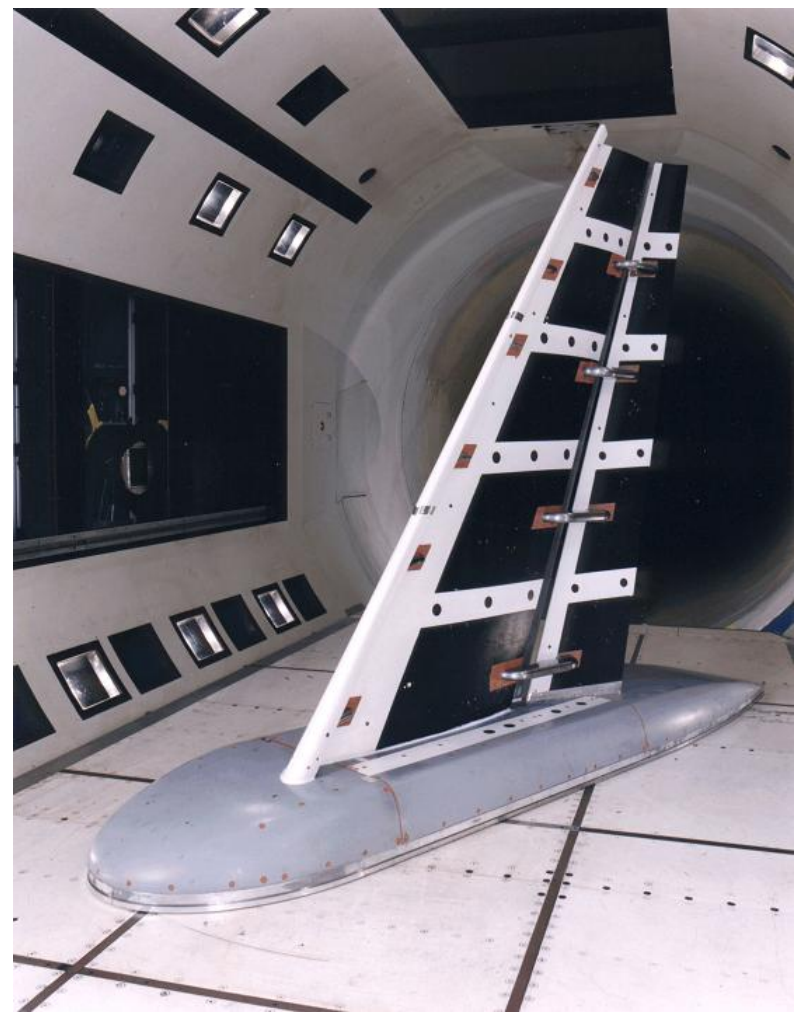

(a) Photo of model in PWT

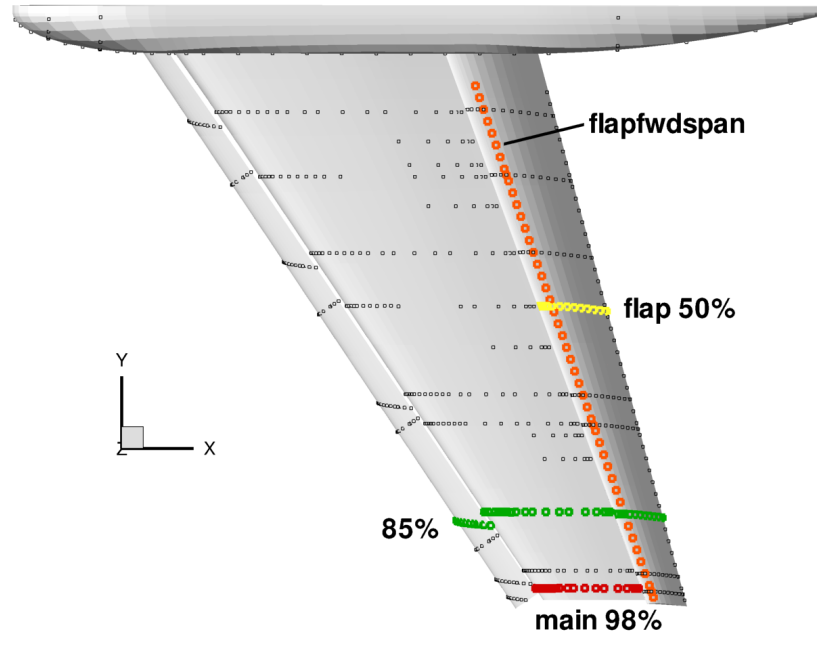

(b) Location of upper surface pressure taps

Figure 1. Trap wing wind tunnel model.

Table 1. Reference geometry for the Trap Wing.

\begin{tabular}{|l|r|}
\hline Mean Aerodynamic Chord $\left(c_{r e f}\right)$ & 39.634 inch \\
\hline Wing Reference Area & $22.028 \mathrm{ft}^{2}$ \\
\hline Wing Span & $85.054 \mathrm{inch}$ \\
\hline Aspect Ratio $(A R)$ & 4.561 \\
\hline Quarter Chord Sweep angle & $29.97 \mathrm{deg}$ \\
\hline
\end{tabular}




\section{CFL3D and FUN3D Non-Adaptive Results}

Several complete sets of runs were made using CFL3D and FUN3D on committee-supplied grids, and were submitted to the HiLiftPW-1 workshop. In addition, a few new runs (not a complete submission) on a grid with brackets created by participants from JAXA were made using FUN3D for this paper. All of the non-adaptive runs are summarized in Table 2. The FUN3D results on tetrahedral grids NCTetA were made as a preliminary step toward computing adaptive results, as described in the next section. Details about the specific grids are not provided here, but documentation can be found in Slotnick et al. ${ }^{32}$ or on the HiLiftPW-1 website.

Table 2. Summary of non-adaptive runs.

\begin{tabular}{llllll}
\hline Code & Full Grid Name & $\begin{array}{l}\text { Abbreviated } \\
\text { Grid Name }\end{array}$ & $\begin{array}{l}\text { Turbulence } \\
\text { Model }\end{array}$ & Brackets? & $\begin{array}{l}\text { Viscous Term } \\
\text { Treatment }\end{array}$ \\
\hline CFL3D & Str-OnetoOne-A & OnetoOneA & SST & No & thin-layer* \\
CFL3D & Str-OnetoOne-A & OnetoOneA & SA & No & thin-layer* \\
CFL3D & Str-OnetoOne-B & OnetoOneB & SST & No & thin-layer*+ \\
CFL3D & Str-OnetoOne-A & OnetoOneA & SA & No & full N-S \\
FUN3D & Unst-Tet-Nodecentered-A & NCTetA & SA & No & full N-S \\
FUN3D & Unst-Mixed-FromTet- & Mix(NCTetA) & SA & No & full N-S \\
& Nodecentered-A & & & & \\
FUN3D & Unst-Mixed-Nodecentered-C & NCMixC & SA & Yes & full N-S \\
\hline
\end{tabular}

$*$ = full N-S with viscous cross-derivatives neglected

$+=$ results not included in this paper

Many workshop participants noted a sensitivity of their solutions to the initial conditions of the computation, especially at the higher angles of attack. The current authors also found that initializing the computation with free-stream conditions could result in a solution with stall characteristics at lower angles of attack than a solution that was initialized from a previously converged computation at a lower angle of attack. Multiple solutions sometimes occurred at the higher angles near stall. The results presented in this paper represent the solution sets that produced higher stall angles.

At the workshop, the CFL3D results presented using SST on the OnetoOneB medium grid stalled at a lower angle of attack than the solutions using the OnetoOneA medium grid. It was recently noted that all of the OnetoOneB computations were initialized with free-stream conditions even at the higher angles of attack near stall. Since the effect of initial conditions on the OnetoOneB grid solutions were not quantified, OnetoOneB results will not be included in this paper.

\section{VI.A. Grid Convergence Study (Case 1) Forces and Moments}

The effects of grid refinement were explored for Configuration 1 at $\alpha=13^{\circ}$ and $28^{\circ}$. Fig. 2 shows $C_{L}, C_{D}$, and $C_{M}$ as a function of $N^{-2 / 3}$, where $N$ represents the number of grid points (for a node-centered scheme) or grid cells (for a cell-centered scheme). On sufficiently refined grids that lie in the asymptotic range of grid convergence, a spatially second-order method should produce a straight line when plotted in this manner. As seen in the figure, the behaviors were not always linear. The SA results with different codes and grids tended to come together to within about 0.05 in $C_{L}, 0.005$ in $C_{D}$, and 0.015 in $C_{M}$ as the grid was refined for $\alpha=13^{\circ}$. The spread was much larger for $\alpha=28^{\circ}$. Experimental values of the forces and moments interpolated to these flow conditions are shown as a filled dot on the $y$-axis of the individual figures. For the most part, grid refinement improved agreement with experiment.

For the FUN3D solutions, the tetrahedral grid, NCTetA, was more sensitive to grid density than the mixed-element grid base on the same nodes, Mix(NCTetA). For both Mix(NCTetA) and NCTetA, the coarse grids yielded results that appear to be out of the asymptotic range. Use of a different family of mixed-element grids (NCMixC) tended to yield slightly lower $C_{L}$ values than $\operatorname{Mix}(\mathrm{NCTetA})$ despite the fact that the NCMixC grids had a globally higher number of nodes. 


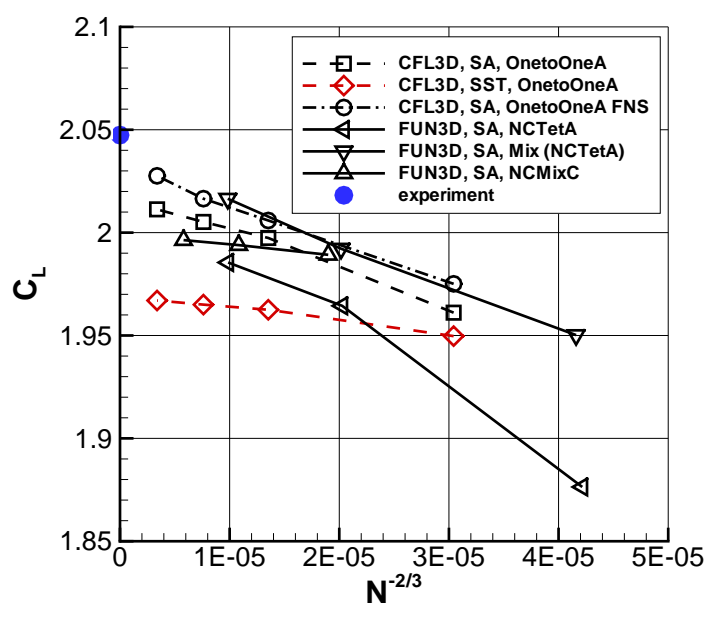

(a) $\alpha=13^{\circ}, C_{L}$

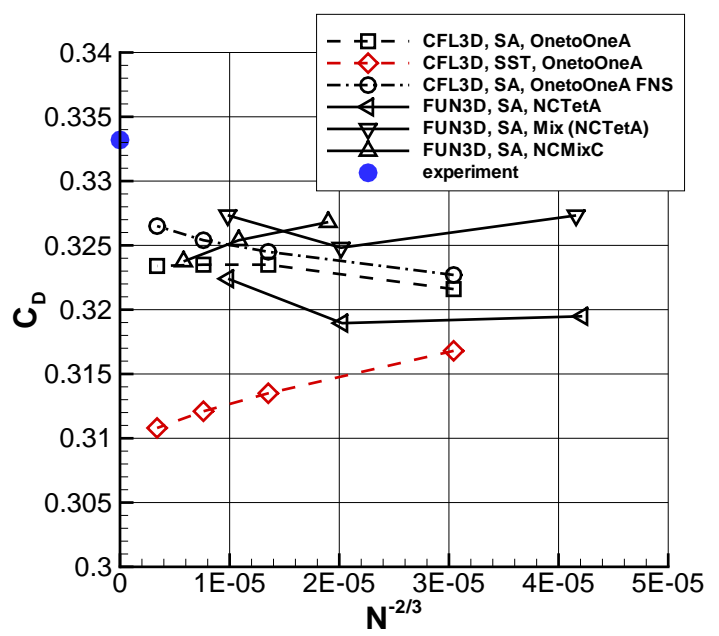

(c) $\alpha=13^{\circ}, C_{D}$

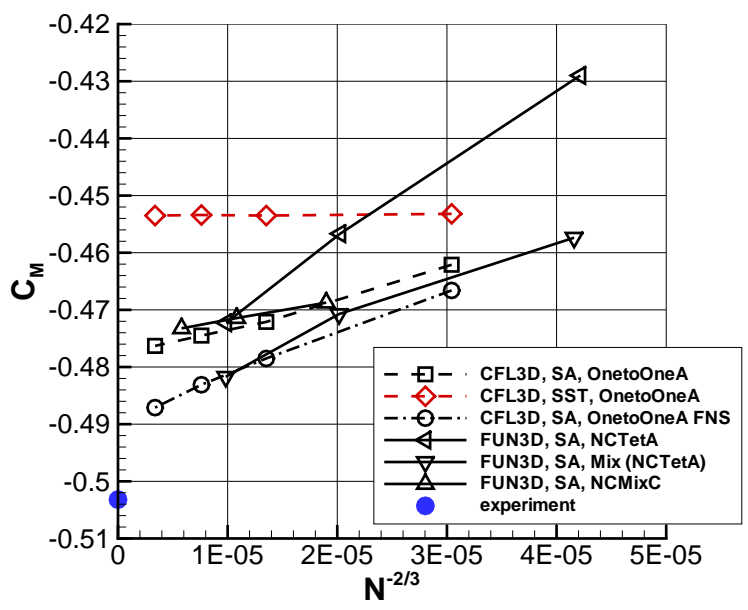

(e) $\alpha=13^{\circ}, C_{M}$

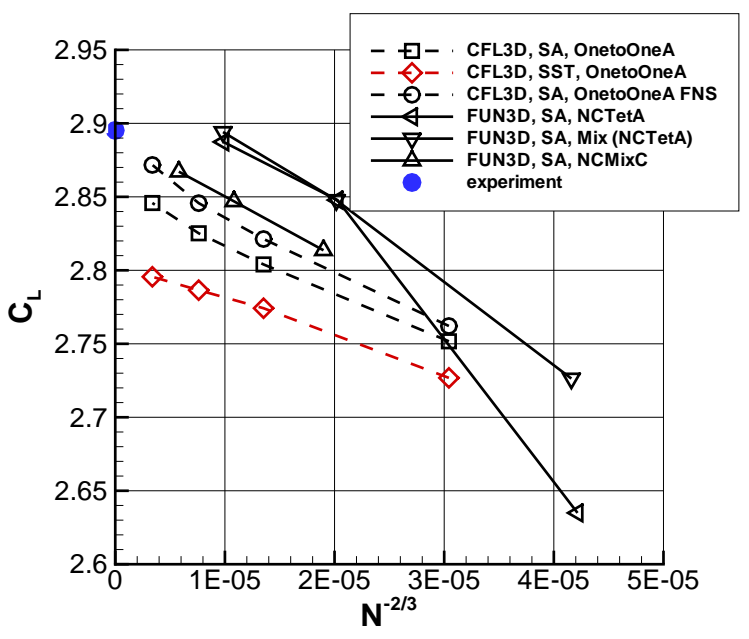

(b) $\alpha=28^{\circ}, C_{L}$

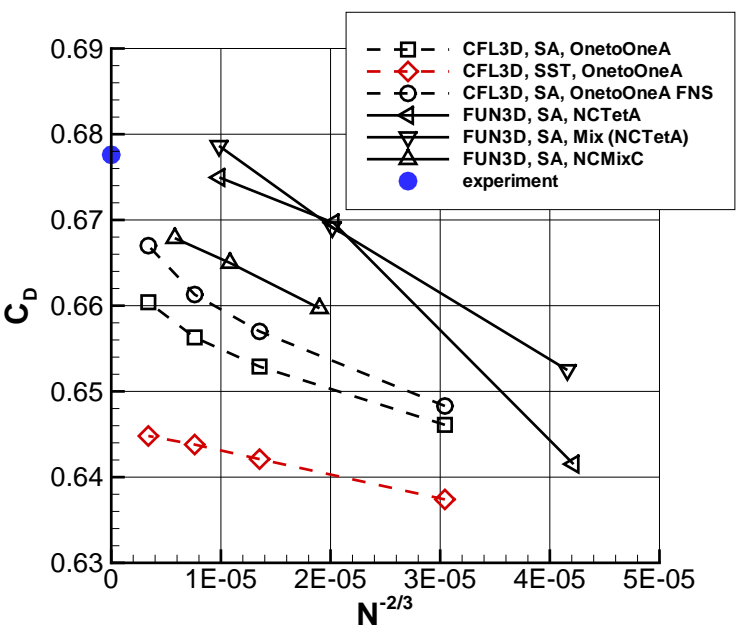

(d) $\alpha=28^{\circ}, C_{D}$

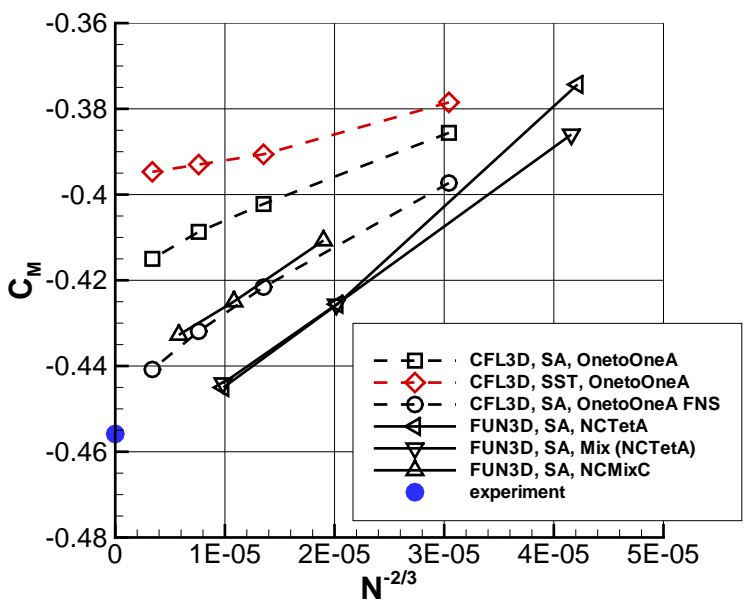

(f) $\alpha=28^{\circ}, C_{M}$

Figure 2. Grid convergence, Configuration 1. 
For the CFL3D solutions, the use of full Navier-Stokes produced higher lift, higher drag, and more negative moment than the approximation that neglected viscous cross-derivatives. Thus the inclusion of the viscous cross-derivatives consistently improved the correlation with the experimental force and moment results. Other effects of neglecting viscous cross-derivative terms will be shown in the next section. The grid convergence study also showed that the CFL3D results using SST converged toward values that were significantly lower in lift, lower in drag, and less negative in moment than the SA results. Further comparisons of the SA and SST turbulence model results will also be shown in the next section.

\section{VI.B. Modeling Sensitivities}

Figure 3 shows the lift curves for all of the CFL3D computations for Configuration 1 on the medium grids. Similar to what was noticed at the workshop by other HiLiftPW-1 participants, computations using the SST model generally were found to yield lower lift levels (in poorer agreement with experiment) than computations using the SA model particularly near $C_{L, \max }$. Typically, SST produced more flap separation than SA. An example is shown in Fig. 4, which shows surface-restricted streamlines for Configuration 1 at $\alpha=13^{\circ}$ using CFL3D. Only SA results will be shown in the remainder of the paper, because the SA model results correlated much better with experiment than the SST results.

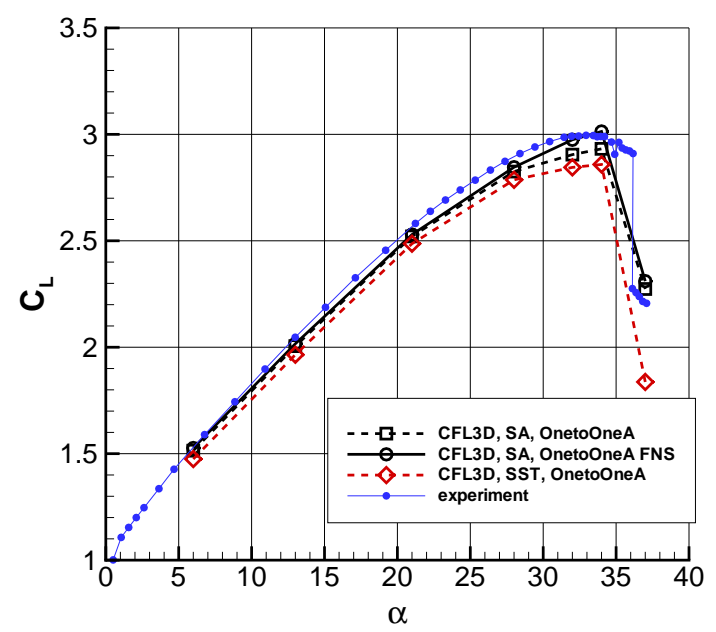

Figure 3. Lift curves for CFL3D computations, Configuration 1, medium grids.

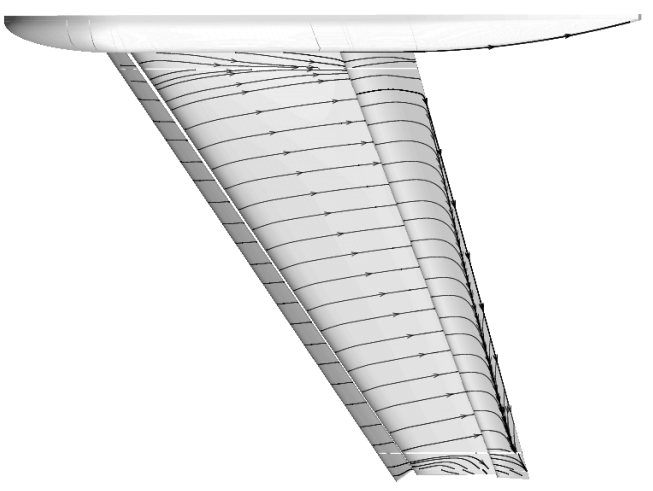

(a) SA

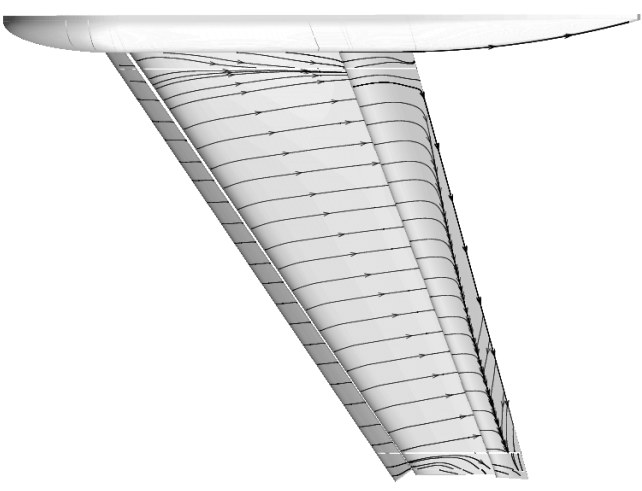

(b) SST

Figure 4. Surface-restricted streamlines, CFL3D, $\alpha=13^{\circ}$, Configuration 1 , medium OnetoOneA grid.

Additionally, Fig. 3 shows some differences between computations that used full Navier-Stokes (FNS) and those that neglected viscous cross-derivative terms: the full Navier-Stokes solutions yielded higher lift levels 
near $C_{L, \max }$. Here, we explore a key reason for the differences. Fig. 5 shows surface pressure coefficients at two locations for Configuration 1 at $\alpha=28^{\circ}$. In the figure, "TLNS" indicates the run with viscous crossderivative terms neglected. At $98 \%$ span location, the TLNS solution yielded dramatically different surface pressures in much poorer agreement with experiment than full Navier-Stokes. The pressure coefficients at the flap forward span location (shown earlier as "flapfwdspan" in Fig. 1(b)) show that the difference occurred primarily in the tip region. Although FNS did not capture the proper $C_{P}$ levels near the wing tip, TLNS was much worse. As will be shown below for the FNS case, there was only a relatively small influence of grid refinement near the wing tip, but the trend was in the correct direction.

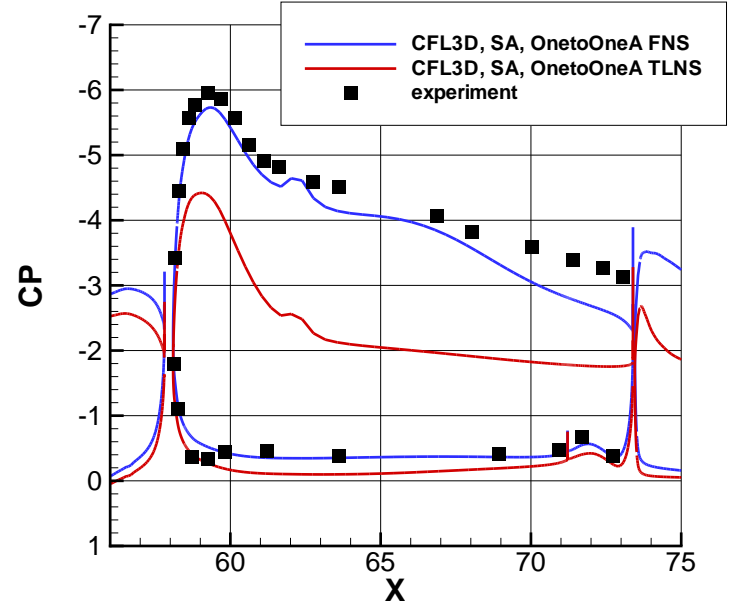

(a) $98 \%$ span station on main element

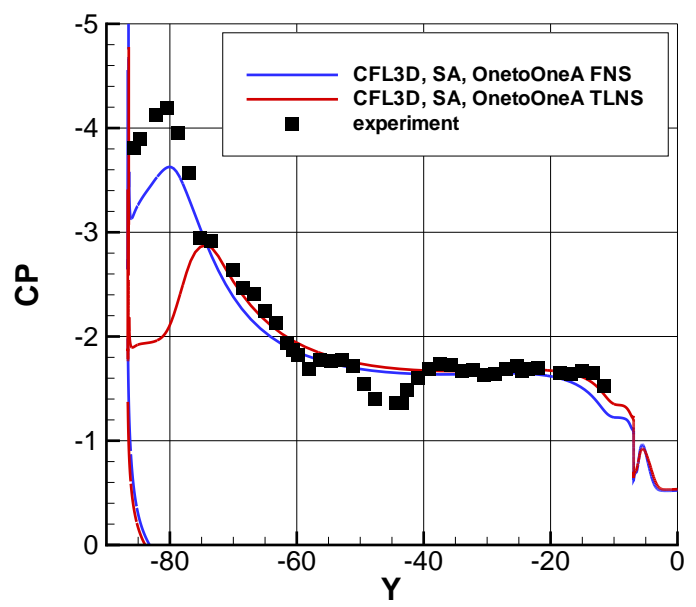

(b) Flap forward span station

Figure 5. Surface pressure coefficients, Configuration 1, $\alpha=28^{\circ}$, CFL3D, SA, fine grid.

Fig. 6 shows vorticity contours at 4 different $x$-planes as a way to visualize the tip vortex development (note that small gaps seen in the geometry are due to a plotting package anomaly and are not real). In the TLNS case where the viscous cross-derivative terms were neglected, the tip vortex was more diffuse than the case where full Navier-Stokes was employed. With a more compact tip vortex, the resulting FNS pressures yielded greater suction in the tip region, in better agreement with experimental data. This difference demonstrates that the use of full Navier-Stokes is very important for this configuration; thin-layer-type approximations should be avoided.

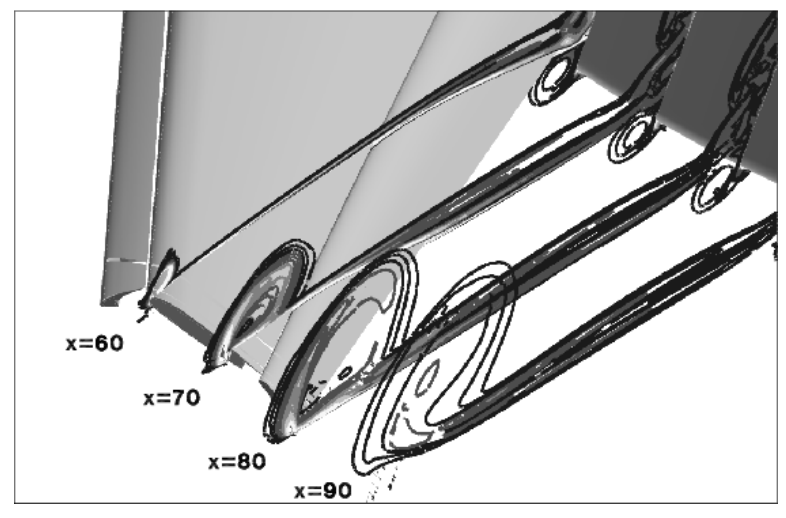

(a) Viscous cross-derivatives neglected

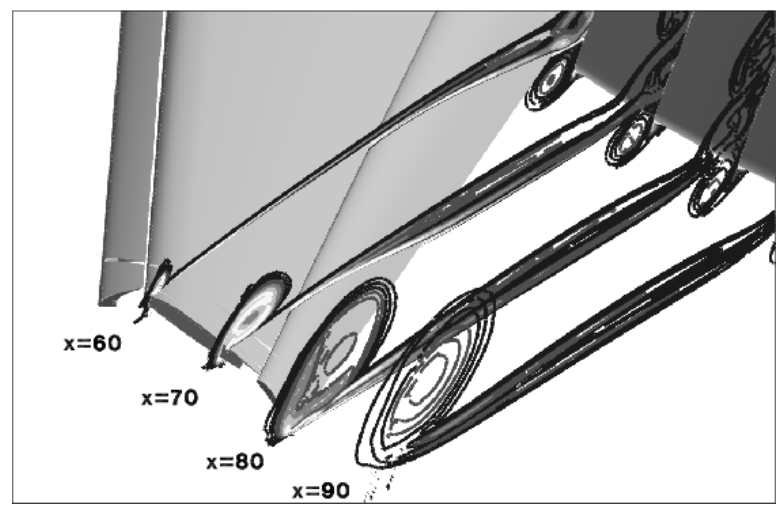

(b) FNS

Figure 6. Vorticity contours showing tip vortex development, CFL3D, Configuration 1, $\alpha=28^{\circ}$, SA, medium OnetoOneA grid. 


\section{VI.C. Slat and Flap Support Effects Study (Case 3)}

The effect of brackets was investigated by using the NCMixC set of grids from JAXA. Only two angles of attack $\left(\alpha=13^{\circ}\right.$ and $\left.28^{\circ}\right)$ were run on medium grids. Computations with brackets yielded lower lift by $\Delta C_{L}=0.0138$ at $\alpha=13^{\circ}$ and by $\Delta C_{L}=0.0519$ at $\alpha=28^{\circ}$ which moves the computational results away from the experimental values. Other workshop participants showed a similar effect for Case 3. Results at the flap forward span station and at the 50\% flap span station are shown in Figs. 7(a) and (b), respectively. With brackets modeled, the pressure coefficients along the flap showed a "scalloping" effect in Fig. 7(a), similar to experimental results. At the $50 \%$ span station on the flap, shown in Fig. 7(b), including brackets yielded a slight improvement in the agreement with experimental surface pressure coefficients. (Note other workshop participants saw a greater improvement in the agreement with experimental surface pressures at this span station.)

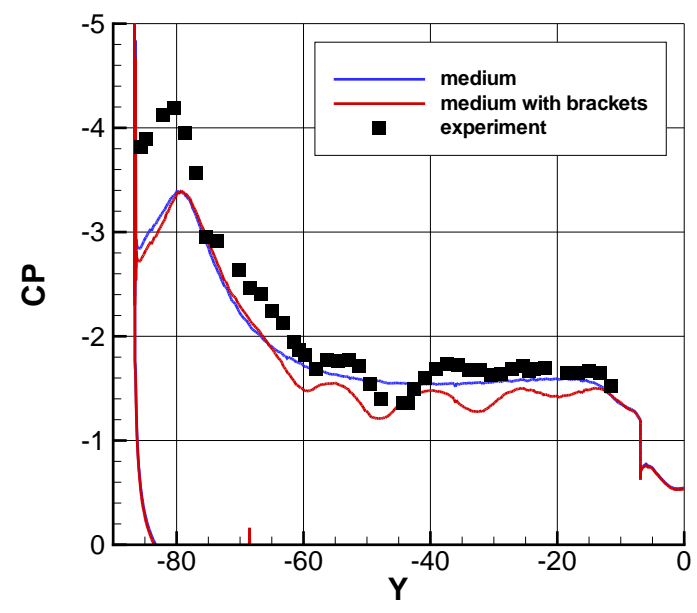

(a) Flap forward span station

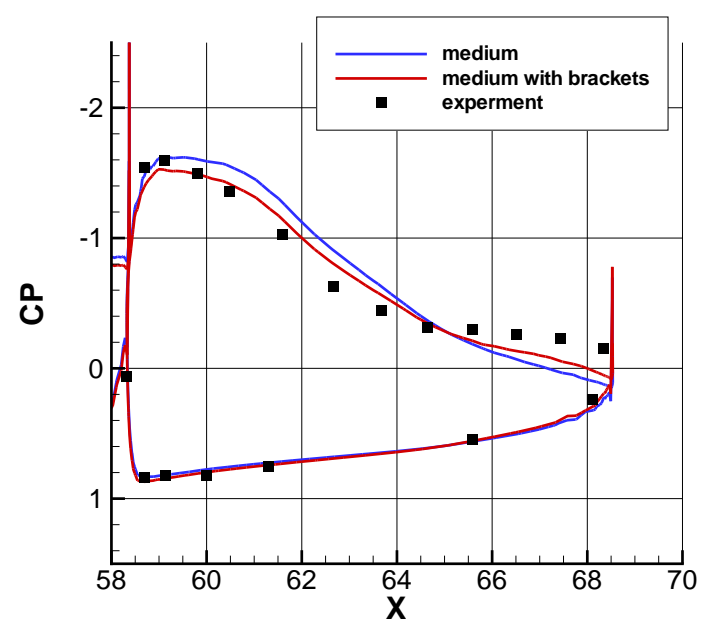

(b) $50 \%$ span location on flap

Figure 7. Surface pressure coefficients showing the effect of brackets, Configuration $1, \alpha=28^{\circ}$, FUN3D, NCMixC grids, SA.

The influence of the brackets on the $\alpha=28^{\circ}$ flowfield behind the wing is shown in Fig. 8. There was some local roll-up of vorticity due to the presence of the brackets. However even for the clean wing, the wake structures tend to diffuse quickly when compared to the structured grid results in Fig. 6, which have a C-mesh topology with inherent wake resolution. The effect of mesh adaptation on the wake structure will be shown in the next section.

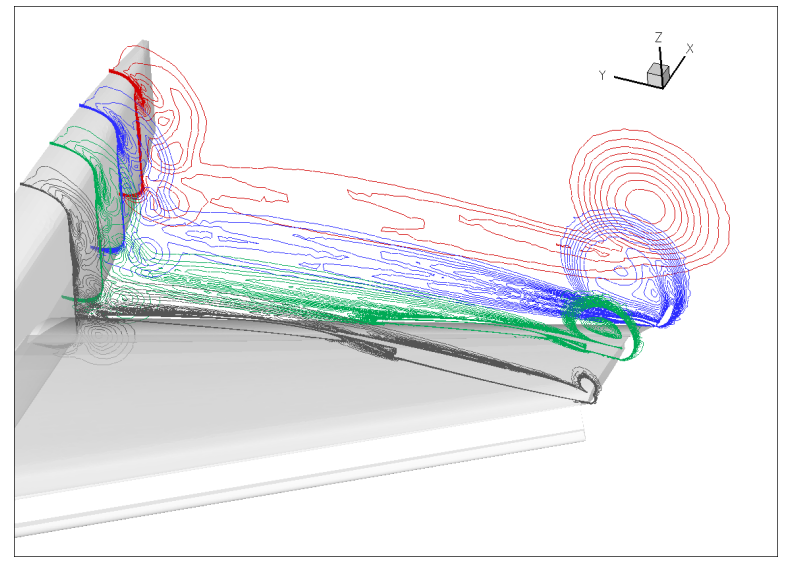

(a) No brackets

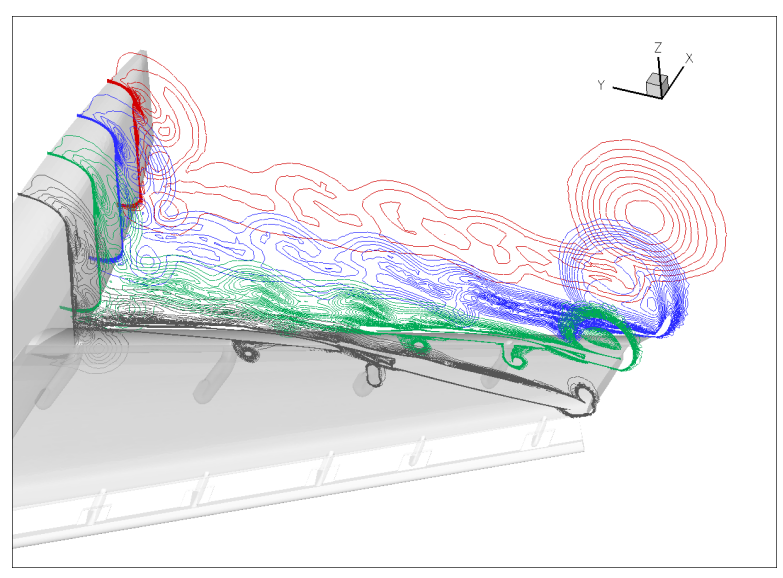

(b) Brackets included

Figure 8. Vorticity contours at $x=60$ "(black), 70" (green), 80"(blue), and 90" (red) constant planes, showing the effect of brackets, Configuration 1, $\alpha=28^{\circ}$, FUN3D, NCMixC grids, SA. 
The effects of both grids and brackets can be visualized by plotting surface restricted streamlines from FUN3D, shown in Fig. 9 for $\alpha=13^{\circ}$. At this angle of attack, the flap trailing edge had separation along much of the span (recall earlier CFL3D results in Fig. 4). Here, the effect of different grids can be seen by comparing Figs. 9(a) and (b). The use of the NCMixC grid produced somewhat less flap separation than use of the $\operatorname{Mix}(\mathrm{NCTetA})$ grid. The reason for this difference is not known. Comparing Figs. 9(b) and (c), it can be seen that the presence of the brackets caused significant modulation of the flap separation line. The effect of flap angle is studied in the next section.

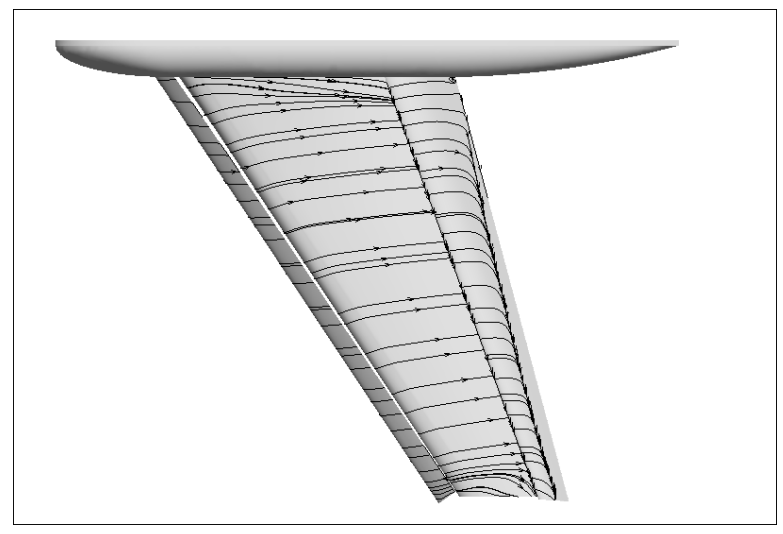

(a) $\operatorname{Mix}(\mathrm{NCTet} A)$ grid, No brackets

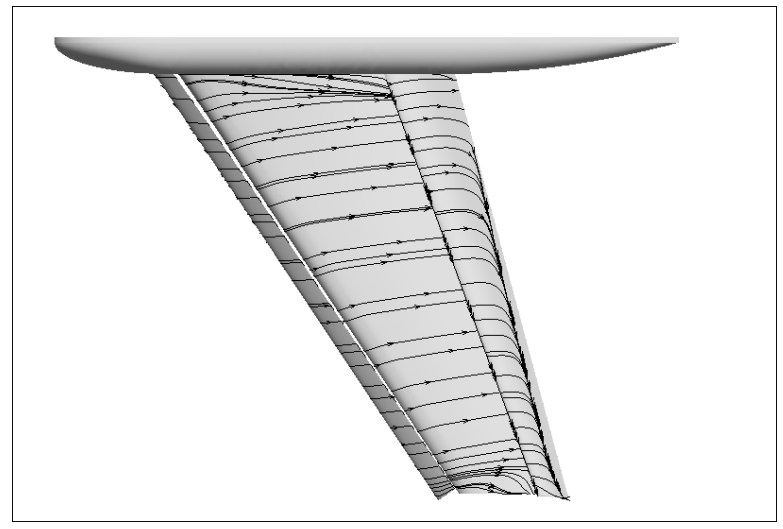

(b) NCMixC grid, No brackets

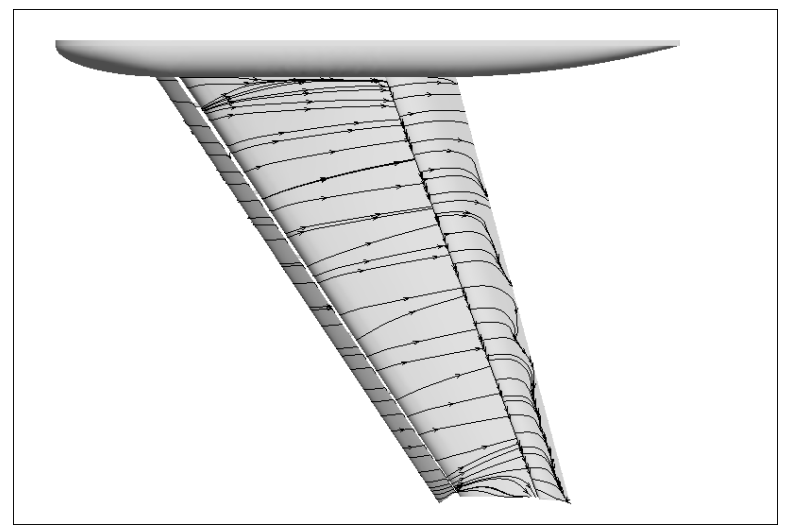

(c) NCMixC grid, Brackets included

Figure 9. Surface restricted streamlines showing the effect of grid and brackets, Configuration 1, $\alpha=13^{\circ}$, FUN3D, SA.

\section{VI.D. Flap Deflection Study (Case 2)}

A subset of the runs will be focused on for the remaining portion of this paper based on experience gained from studying the modeling sensitivities. Figure 10 shows lift curves, drag polars, and moment polars for CFL3D on the OnetoOneA grid, FUN3D on the NCTetA grid, and FUN3D on the Mix(NCTetA) grid. All methods shown used the full Navier-Stokes formulation and SA turbulence model. For Case 2, angle of attack sweeps for Configuration 1 and Configuration 8 were performed on the medium grids. The left column shows Configuration 1 and the right column shows Configuration 8. All three sets of computational results were very similar, with the largest differences in the moment coefficient. The lift and drag coefficients were generally predicted slightly low compared to experiment, and the moment coefficient was generally predicted low in magnitude compared to experiment. Experiment measurements are only available at and below $\alpha=28^{\circ}$ for Configuration 8 Recalling Fig. 2, refining the grid tended to bring computed results closer to experimental levels. For Configuration 1, the computations consistently predicted stall between $\alpha=34^{\circ}$ and $\alpha=37^{\circ}$ with the slat stalling first followed by the wing. For Configuration 8, the CFL3D results showed a similar stall behavior, but the FUN3D results on the NCTetA grid stalled at a lower angle. The FUN3D results on the $\operatorname{Mix}(\mathrm{NCTetA})$ grid did not indicate the same type of stall mechanism for Configuration 8 as for Configuration 1. For the FUN3D computation on Mix(NCTetA), the wing stalled first. 


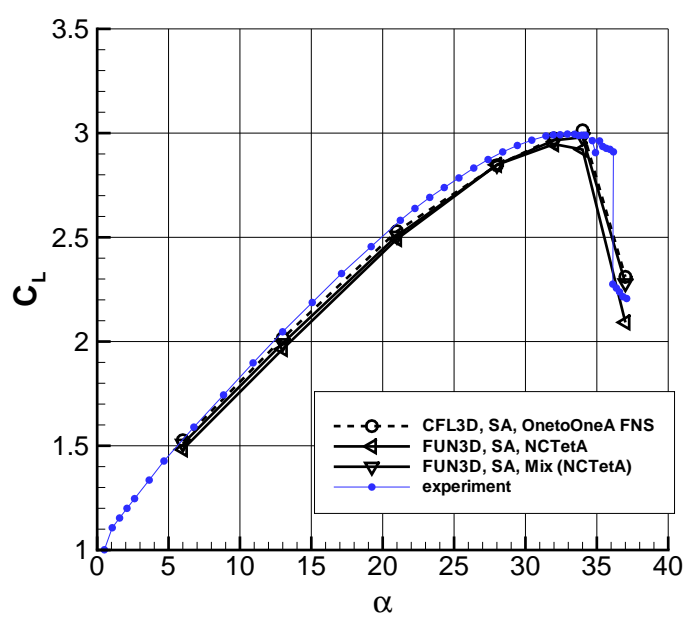

(a) Configuration 1, lift curve

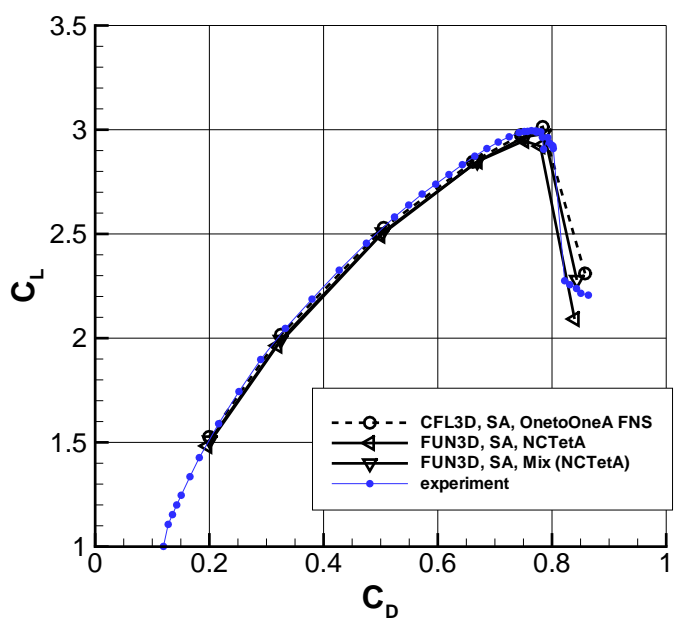

(c) Configuration 1, drag polar

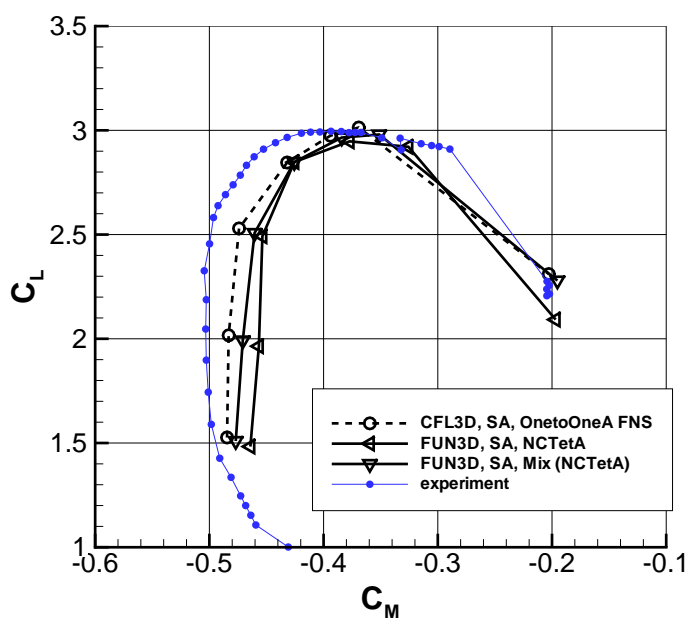

(e) Configuration 1, moment polar

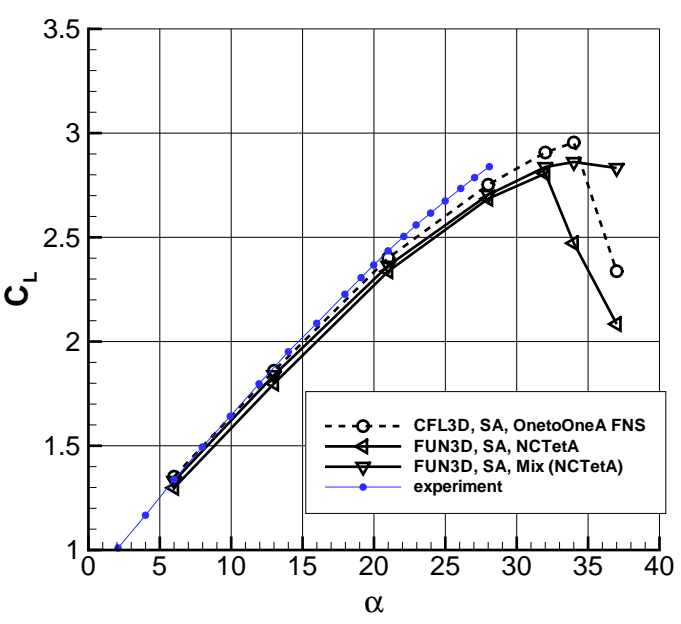

(b) Configuration 8, lift curve

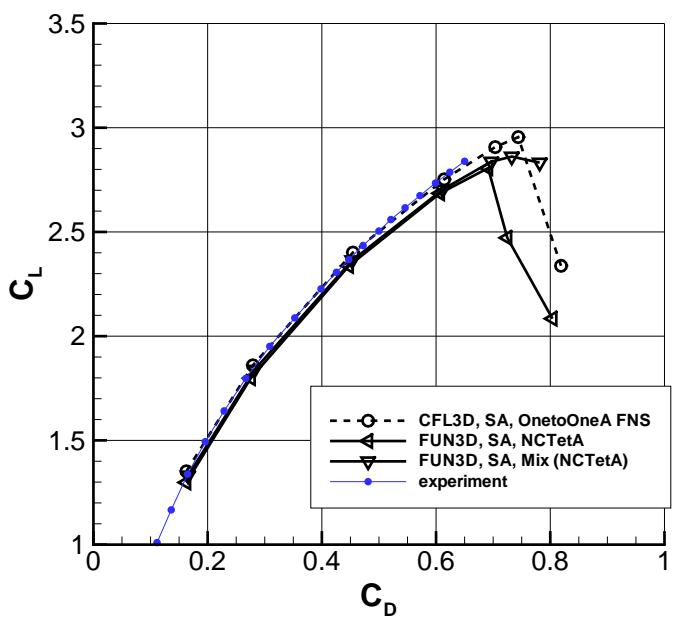

(d) Configuration 8, drag polar

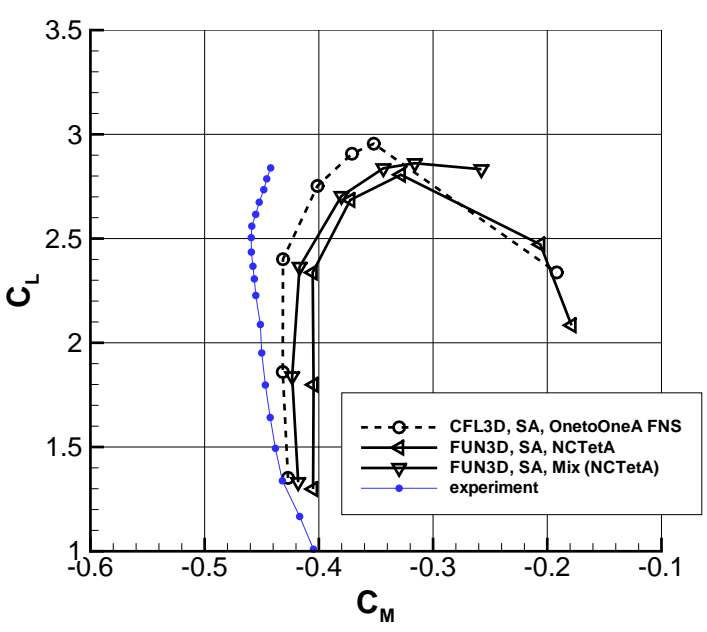

(f) Configuration 8, moment polar

Figure 10. Force and moment curves, medium grids. 
Figure 11 shows the delta force and moment of Configuration 1 minus Configuration 8. The delta lift, drag, and pitching moment curves indicate that the effect of flap deflection was slightly under-predicted by the computations at the lower angles of attack and over-predicted at the higher angles. The CFL3D results were closer to the experimental trends than FUN3D at the higher angles of attack. For the FUN3D results, the inconsistent stall angle prediction between Configuration 1 and Configuration 8 led to an erratic prediction of the flap deflection effect near the stall angle.

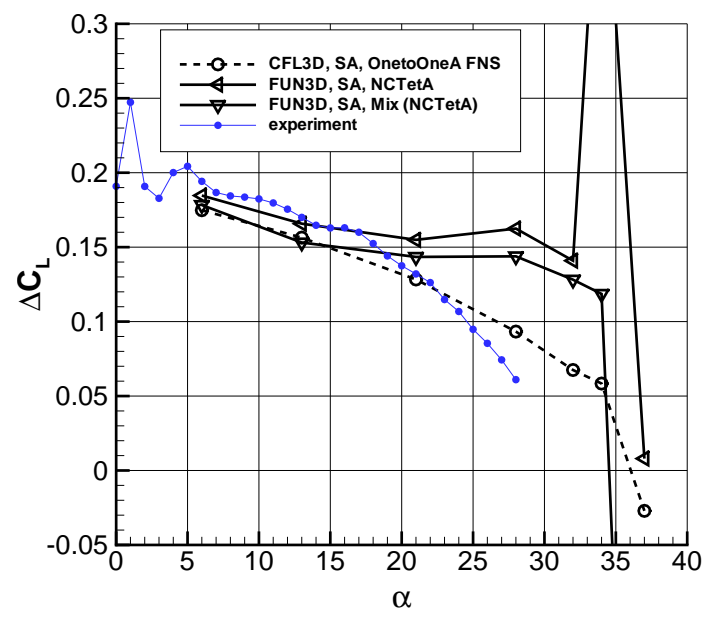

(a) Delta lift curve

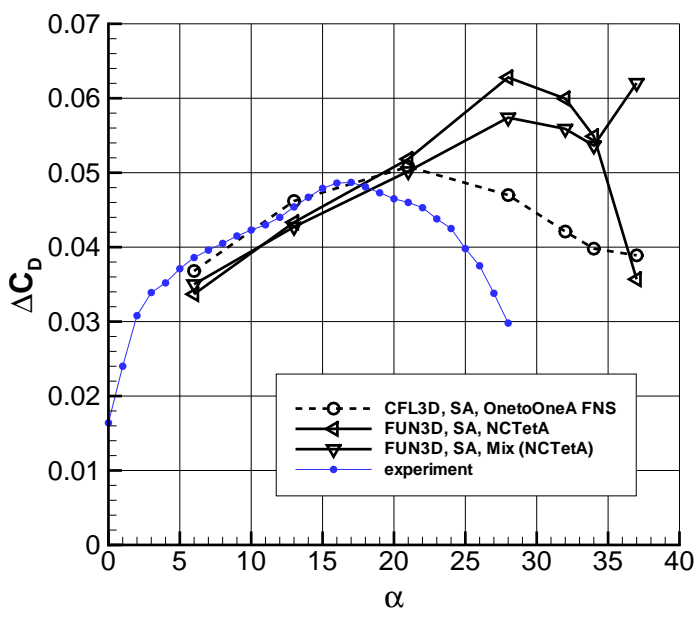

(b) Delta drag curve

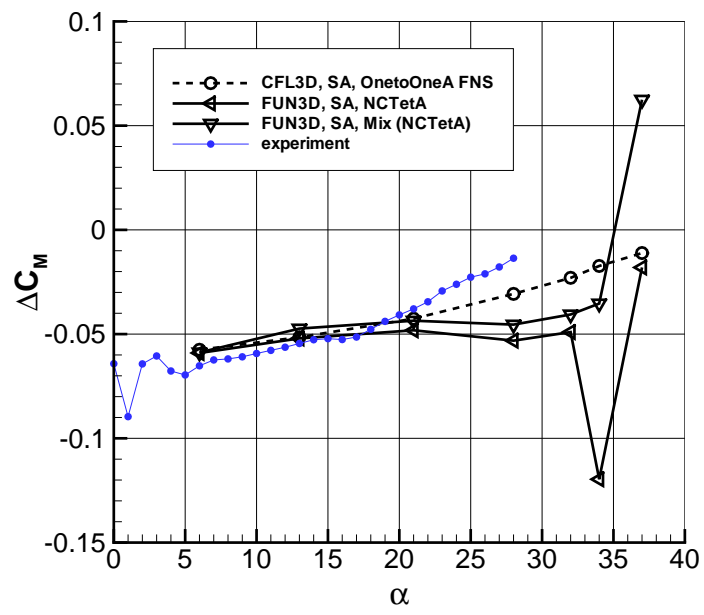

(c) Delta moment curve

Figure 11. Delta force and moment curves, medium grids.

\section{VI.E. Grid Convergence Study (Case 1) Surface Pressures}

Comparing the surface pressures from the grid convergence study illustrate the areas of the flow where the code-to-code and grid-to-grid are significant. The grid convergence of FUN3D and CFL3D surface pressures are shown for the $85 \%$ span station of the flap (Configuration 1 and $\alpha=13^{\circ}$ ) Fig. 12 FUN3D exhibited greater grid sensitivity than CFL3D. The CFL3D variation was localized at the peak suction of the flap. The FUN3D refinement showed increased suction on the forward portion of flap and a change in the flap trailing edge separation characteristic. Although not shown, use of FUN3D with all tetrahedral NCTetA grids yielded even greater grid sensitivity. However, on the finest grid the results overall were very similar. 


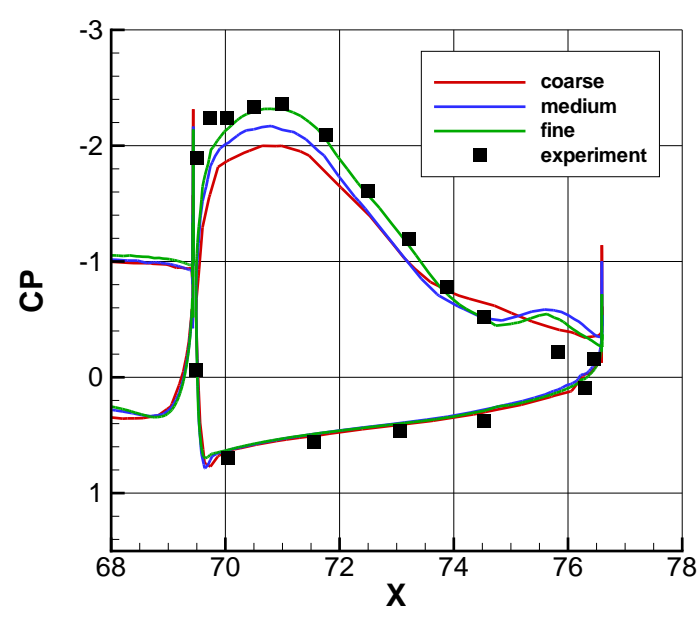

(a) FUN3D on Mix(NCTetA) grids

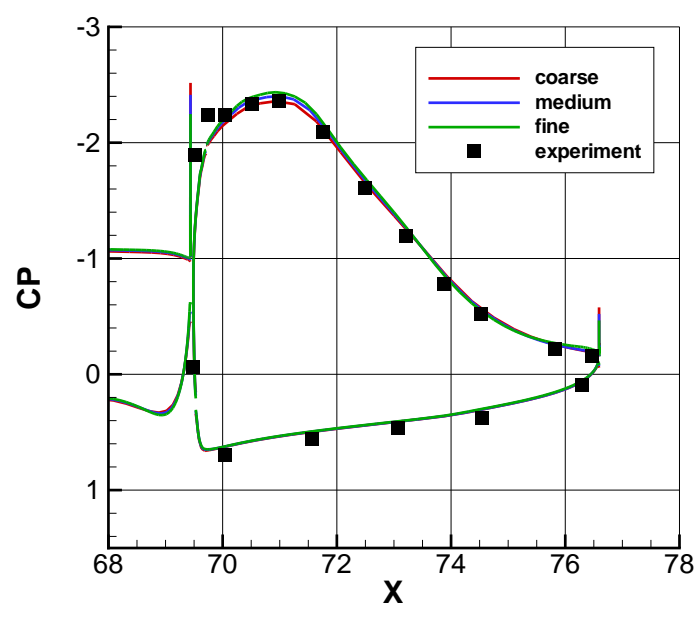

(b) CFL3D on OnetoOneA grids, FNS

Figure 12. Grid convergence of surface pressure coefficients, Configuration $1, \alpha=13^{\circ}, \mathbf{S A}, \mathbf{8 5 \%}$ span station on flap.

The flap forward span station (Configuration 1 and $\alpha=28^{\circ}$ ) is is shown in Fig. 13. As seen in Fig. 1(b), the flap forward span station is near quarter chord of the flap upper surface. Both FUN3D and CFL3D have an improvement in the prediction of the tip vortex with grid refinement. FUN3D exhibited greater variation due to grid refinement in the inner portion of the wing.

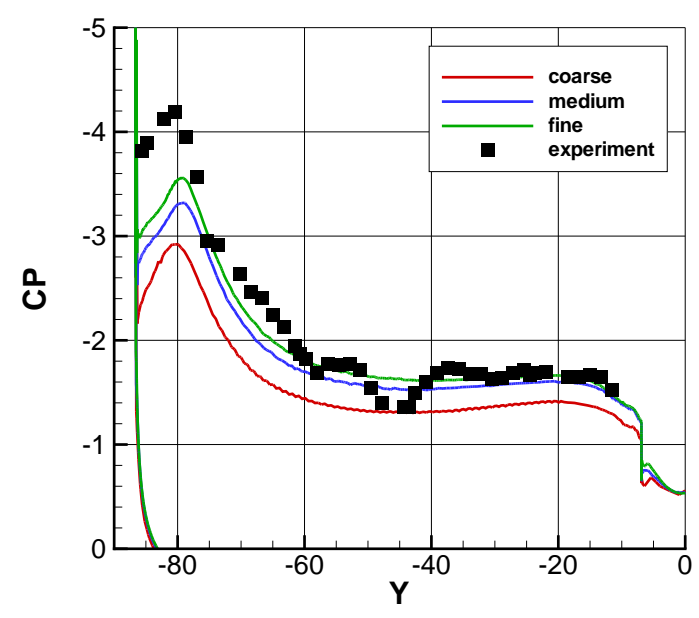

(a) FUN3D on Mix (NCTetA) grids

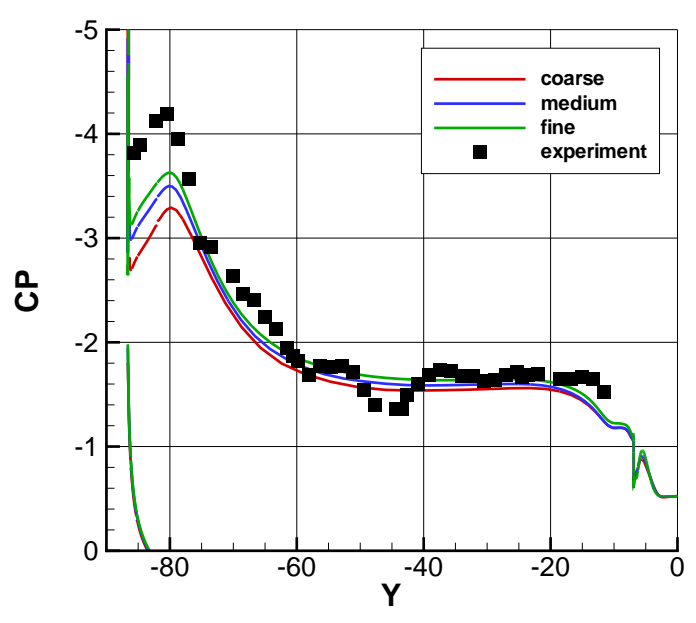

(b) CFL3D on OnetoOneA grids, FNS

Figure 13. Grid convergence of surface pressure coefficients, Configuration 1, $\alpha=28^{\circ}$, SA, flap forward span station.

Fig. 14 compares four different combinations of grid and code on fine grids for Configuration $1, \alpha=13^{\circ}$, at the $85 \%$ span station on all elements. Results were almost indistinguishable on the slat and main elements, but FUN3D produced slightly less upper surface suction and a greater amount of trailing edge separation on the flap than CFL3D. The effect of adaptive mesh refinement on this latter characteristic will be explored in the next section.

Similarly, Fig. 15 compares the Configuration 1, $\alpha=28^{\circ}$, fine grid results for the $85 \%$ span station on all elements. At the $85 \%$ span station, all four sets of results on fine grids showed only minor differences with the greatest variation in the flap upper surface peak pressure. In Fig. 15(c), all of the CFD predictions had a lower pressure on the flap lower surface than the measurements. The reason for this discrepancy is not known. 


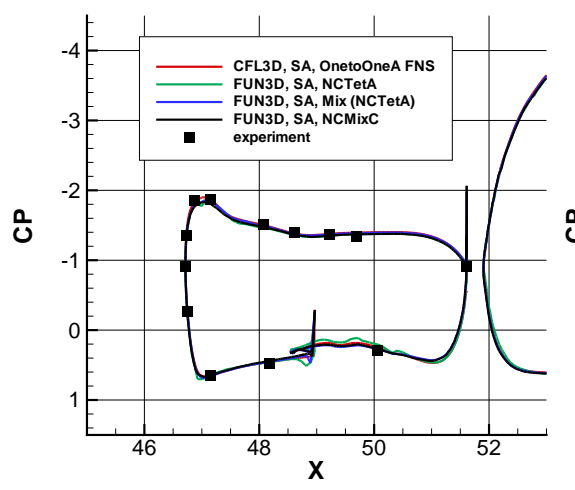

(a) Slat

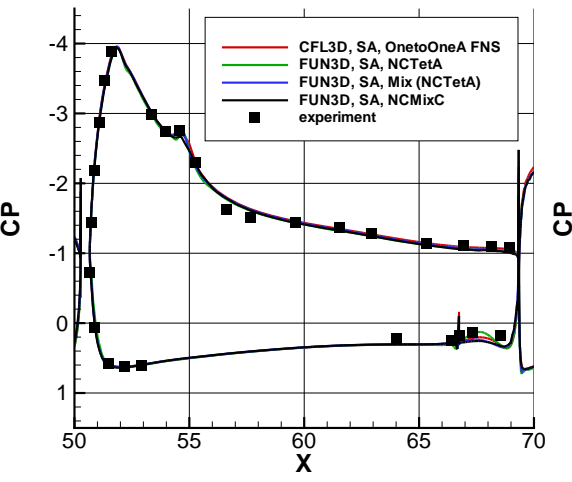

(b) Main

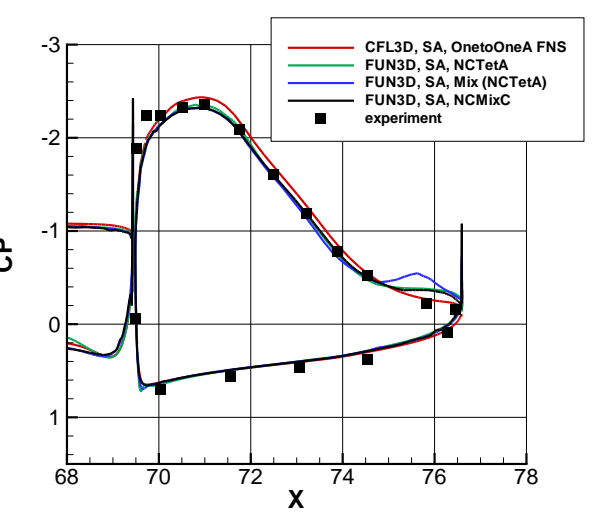

(c) Flap

Figure 14. Surface pressure coefficients, Configuration 1, $\alpha=13^{\circ}, \mathbf{S A}, \mathbf{8 5 \%}$ span station on all elements, fine grids.

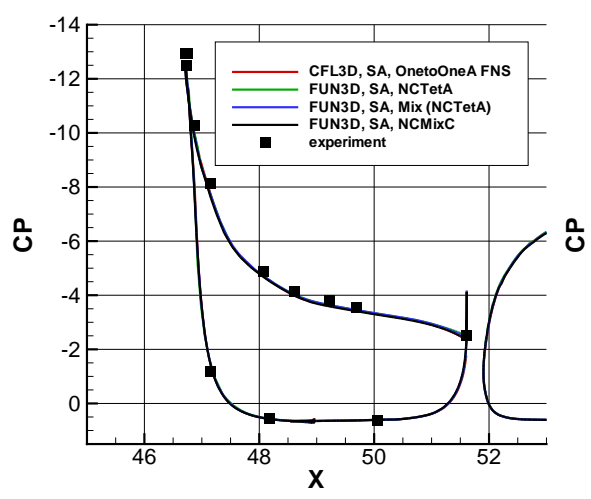

(a) Slat

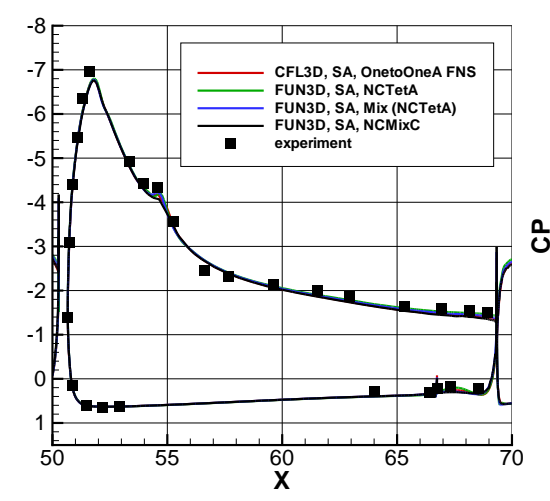

(b) Main

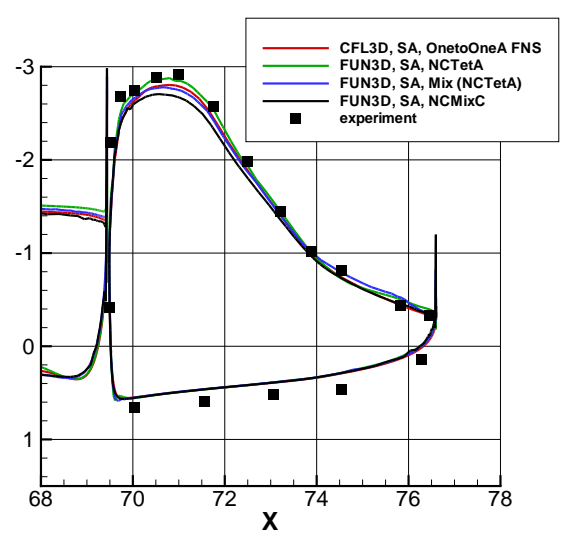

(c) Flap

Figure 15. Surface pressure coefficients, Configuration 1, $\alpha=28^{\circ}, \mathbf{S A}, 85 \%$ span station on all elements, fine grids. 
A similar comparison of fine results at the flap forward span station is also shown in Fig. 16. There is a slight variation in pressure coefficient results across the flap span with the tip and root regions showing the greatest variation. All of the computations predict a lower peak-suction value near the tip region than measured in the experiment.

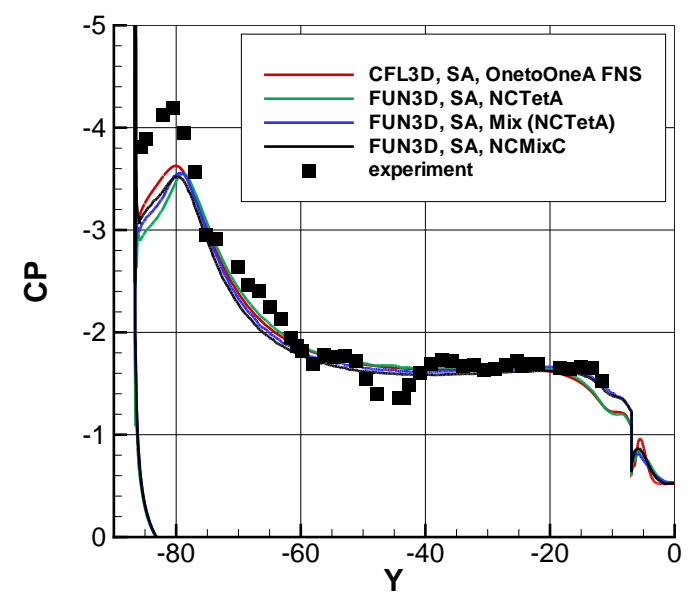

Figure 16. Surface pressure coefficients, Configuration 1, $\alpha=28^{\circ}$, SA, flap forward span station, fine grids.

\section{FUN3D Adaptive Results}

Each of the adapted cases use a converged solution on the original grid at that angle of attack. These original grid solutions were computed with the aid of angle of attack sequencing; each angle of attack solution was restarted from a converged lower angle of attack solution on the same original grid. Attempts to start the high angle of attack cases from freestream initial conditions (not shown) were problematic. These high- $\alpha$ cases started from freestream either failed to converged or appeared to converge to a condition with massive separation and lower coefficient of lift than the $\alpha$-sequenced cases. Adaptation on these massively separated solutions did not reattach the flow or reach a significantly higher coefficient of lift. Angle of attack sequencing was a practice employed by many workshop participants.

Coefficient of lift is plotted as a function of coefficient of drag for the workshop-provided coarse and medium node-based tetrahedral Configuration 1 grids in Fig. 17(a). The original grid is show as open symbols at the workshop-specified angles of attack. Each of the original grids was adapted and the results are shown as filled symbols, Fig. 17(a). The adapted coarse grids used the embedded-grid error estimate and the adapted medium grids used the single-grid error estimate. The single-grid error estimate adaptation included constant complexity metric scaling, Eq. (2). Adaptation with the embedded- and single-grid error estimates is compared for the medium grid at $\alpha=13^{\circ}$ in the following sections. The adapted coarse grid results approximated the original medium grid results at $\alpha=28^{\circ}$ and below. The maximum lift was underpredicted by the original and adapted coarse grid computations. When looking at the entire polar, there was little difference between the original and adapted medium grid results. Drag and lift were lower for the high- $\alpha$ pre-stall adapted grids, but were on the same curve as the original grids.

\section{VII.A. Coarse Grid Embedded-Grid Error Estimate Adaptation}

A zoom of the lift-drag polar at $\alpha=13^{\circ}$ is shown in Fig. 17(b). The coarse (3.6M nodes), medium (11M nodes), and fine (32M nodes) original grids are shown as the open circles. The coarse and medium original grids were used for adaptation with the embedded-grid error estimate (filled square) and the single-grid error estimate (open squares). The series of adapted grids are connected by solid lines, forming a trajectory. The script driving the adaptive processes begins by restarting the flow solver, so the first point of each adaptive trajectory is at a slightly different location than the uniform refinement points due to different iterative convergence levels. The embedded-grid error estimate adaption based on the coarse original grid 


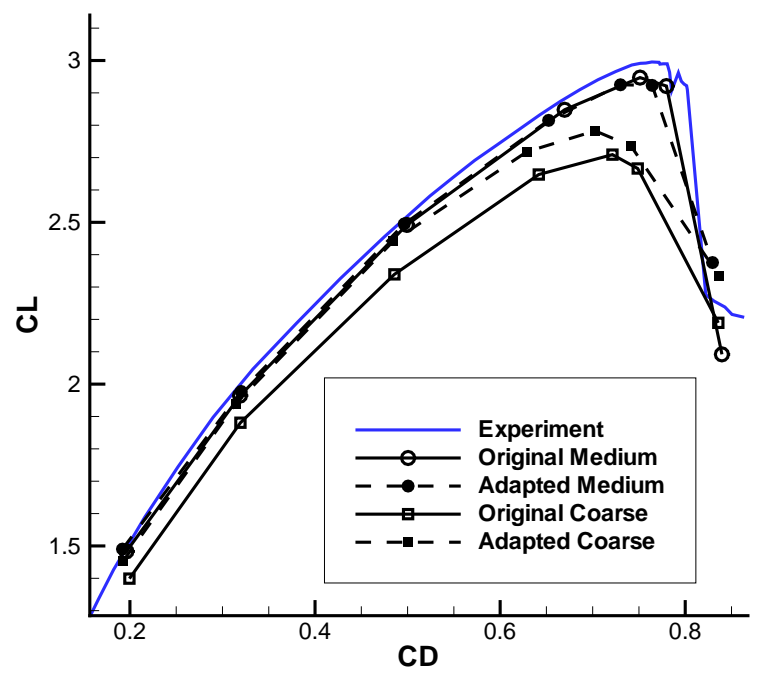

(a) Entire polar.

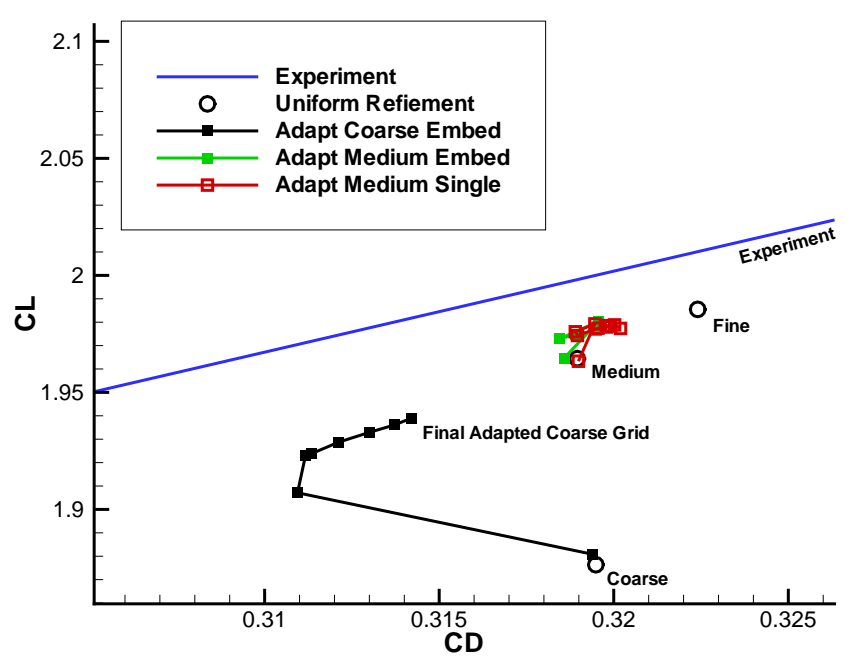

(b) Zoom of adaptive trajectory, $\alpha=13^{\circ}$.

Figure 17. Lift as a function of drag, Configuration 1.

is approaching the original medium grid point. The final adapted coarse grid has $11 \mathrm{M}$ nodes. The medium grid adaptations, which are shown in more detail in the next section, also trend toward the fine grid point. Output-based adaptation is formulated to address discretization error and not modeling error, so it should tend toward the uniformly-refined grid answer and not the experimental measurements when modeling errors are present.

The original and final adapted volume tetrahedral grid for the coarse grid adaptation at $\alpha=13^{\circ}$ is sliced at the $85 \%$ span station, Fig. 18. The intersection of a plane and the tetrahedral elements is shown for the original, Fig. 18(a), and adapted, Fig. 18(b), grids. The resolution of the slat, main element, and flap stagnation stream lines has been improved. The upper surface of the main element and wakes have been resolved with anisotropic elements. The $u$-velocity at the $85 \%$ span station is shown for the original, Fig. 18(c), and adapted, Fig. 18(d), grids. The velocity deficit in the wake is better defined for the adapted grid. The adapted slat and flap gap regions are detailed in the next figures.

The slat gap region at $\alpha=13^{\circ}$ is sliced at the $85 \%$ span station, Fig. 19. The adapted grid, Fig. 19(b), is more refined in the slat stagnation, gap, and wake regions than the original grid, Fig. 19(a). The region of frozen boundary layer grid can be seen close to the wing in Fig. 19(b). The slat gap jet has a more defined boundary in the adapted grid, Fig. 19(d). The slat wake velocity deficit is also better defined and propagated a longer distance over the main element in the adapted grid, Fig. 19(d), than the original grid, Fig. 19(c). The flow has greater acceleration above the slat wake as compared to the original coarse grid solution.

The flap gap region at $\alpha=13^{\circ}$ is sliced at the $85 \%$ span station, Fig. 20. The adapted grid, Fig. 20(b), is more refined near the flap stagnation region, gap, upper surface, and wake. The adapted grid is also more refined near the main element lower surface, cove, and upper surface. The region of frozen boundary layer grid can also be seen close to the wing in Fig. 20(b). The peak velocity in the flap gap jet increases for the adaptive grid as well as the region above the main element wake. The region of strongly reversed flow above the aft portion of the flap (large negative values of $u$-velocity) is decreased in the adaptive grid. These trends are also indicated by the grid convergence of the surface pressure coefficients for the Mix(NCTetA) grids, Fig. 12(a). With grid refinement, the peak negative pressure coefficients increases indicating a higher velocity at $x=71$ and the region of separated flow is decreased at $x=76$.

\section{VII.B. Medium Grid Single- and Embedded-Grid Error Estimate Adaptations}

Details of the medium grid adaptive trajectories are provided in Fig. 21(a) as a closer zoom of Fig. 17(b). The adaptive trajectories for the single- and embedded-grid error estimate were similar. Due to the resources required for the embedded-grid error estimate, this is the only case performed to compare the single- and embedded-grid error estimates. The final embedded-grid adaptation is $17 \mathrm{M}$ nodes and the final single grid 


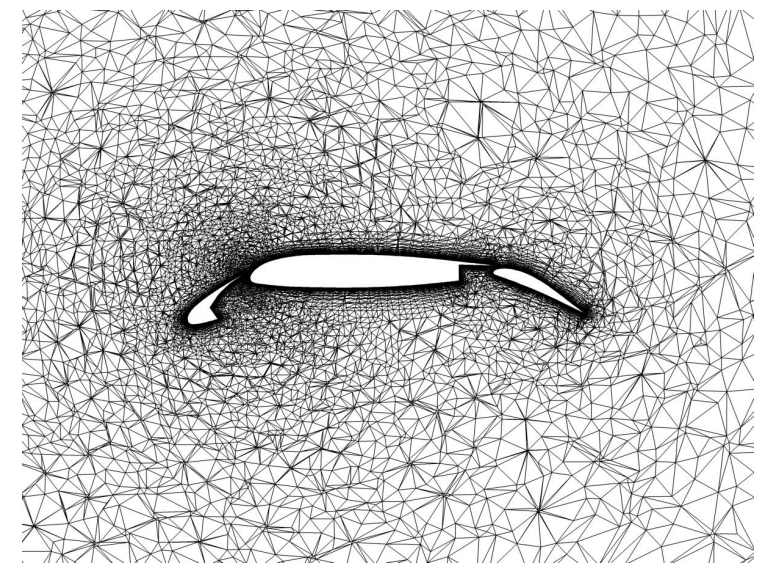

(a) Original coarse grid (3.6M nodes).

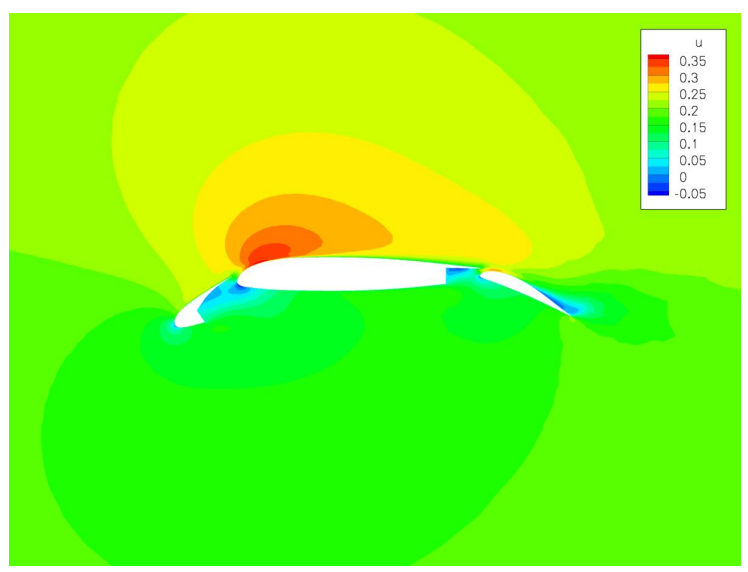

(c) Original coarse grid $u$-velocity.

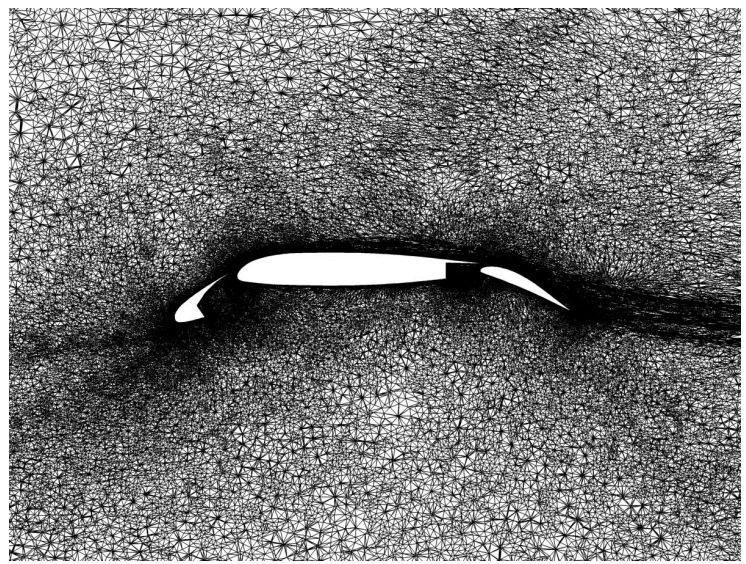

(b) Final adapted coarse grid (11.0M nodes).

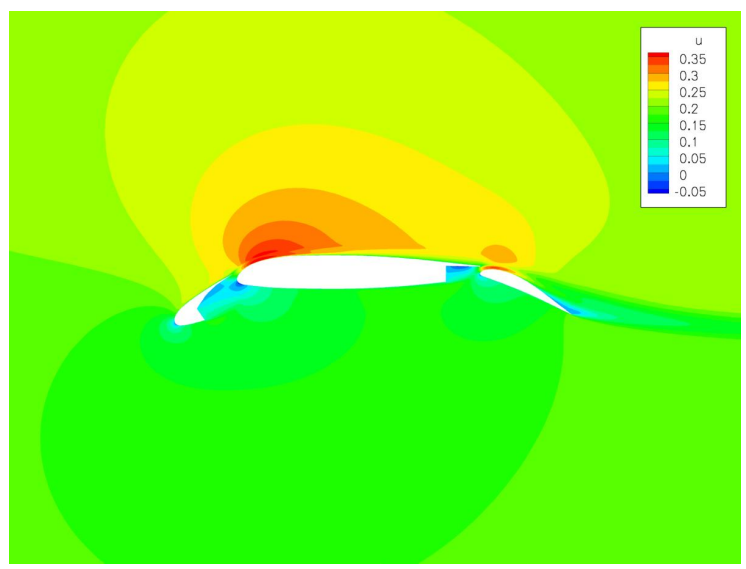

(d) Final adapted coarse grid $u$-velocity.

Figure 18. Slice of volume grid at the $85 \%$ span station for the original and adapted coarse grid, Configuration 1 , $\alpha=13^{\circ}$. 


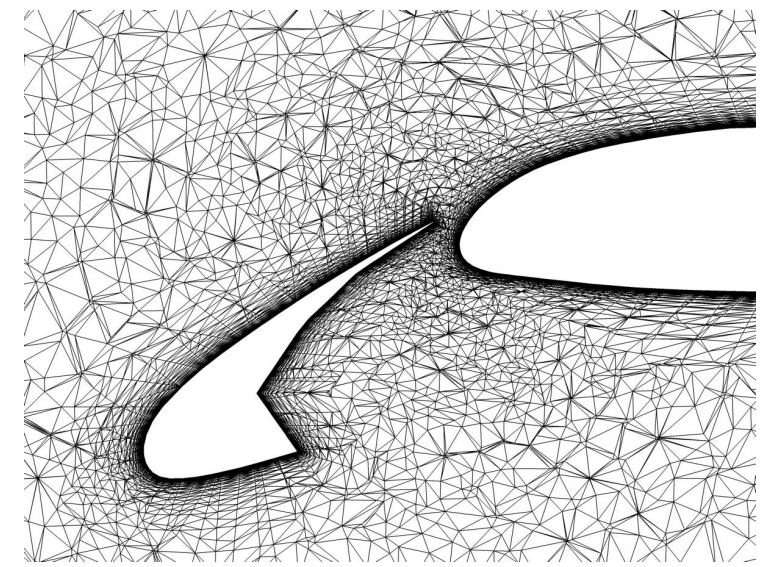

(a) Original coarse grid (3.6M nodes).

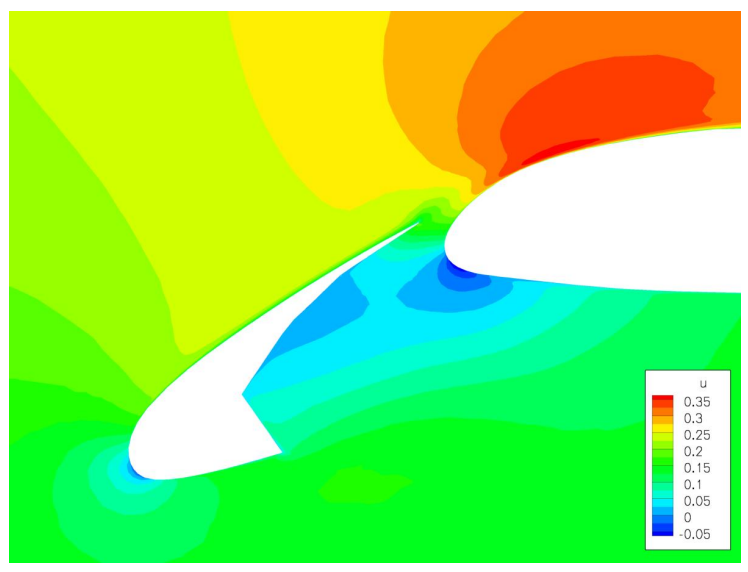

(c) Original coarse grid $u$-velocity.

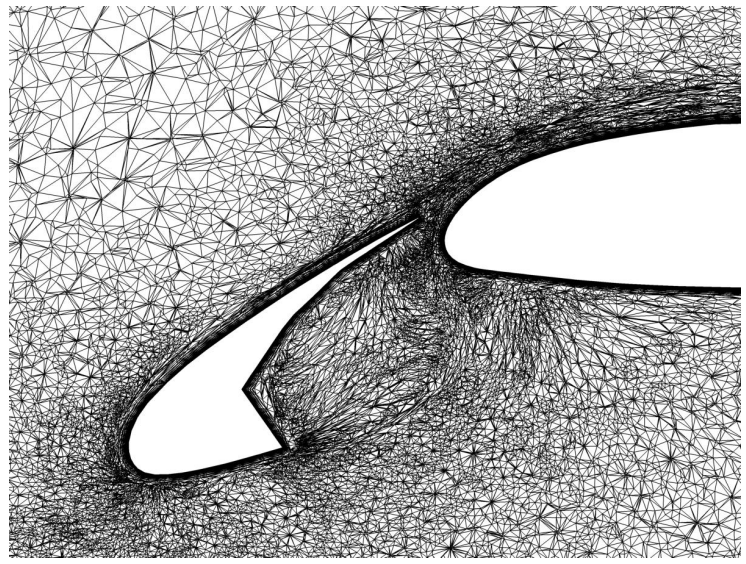

(b) Final adapted coarse grid (11.0M nodes).

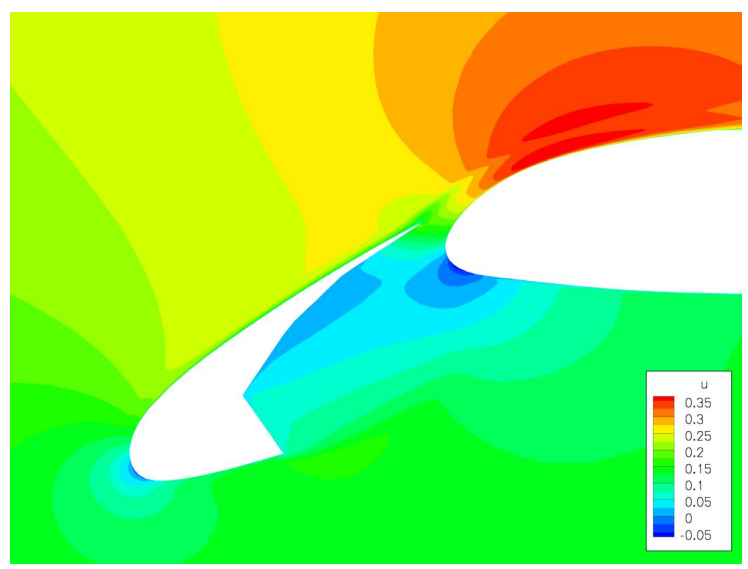

(d) Final adapted coarse grid $u$-velocity.

Figure 19. Slice of volume grid at the $85 \%$ span station for the slat of the original and adapted coarse grid, Configuration $1, \alpha=13^{\circ}$. 


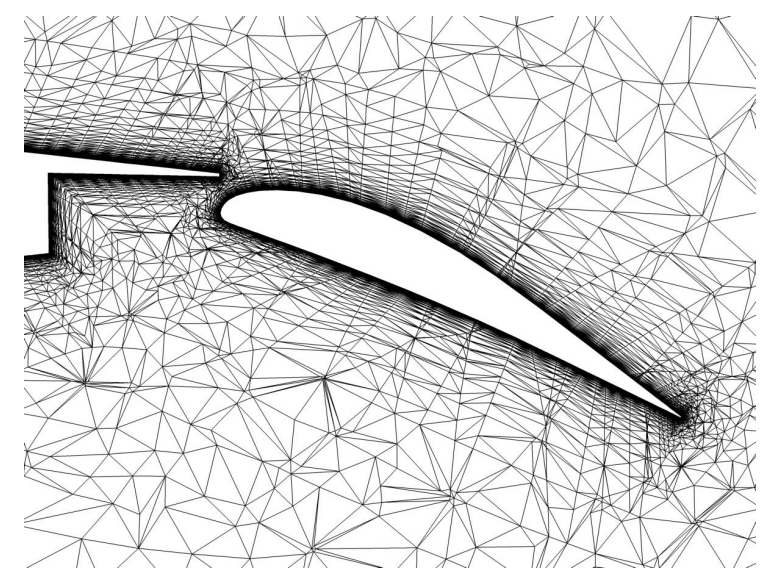

(a) Original coarse grid (3.6M nodes).

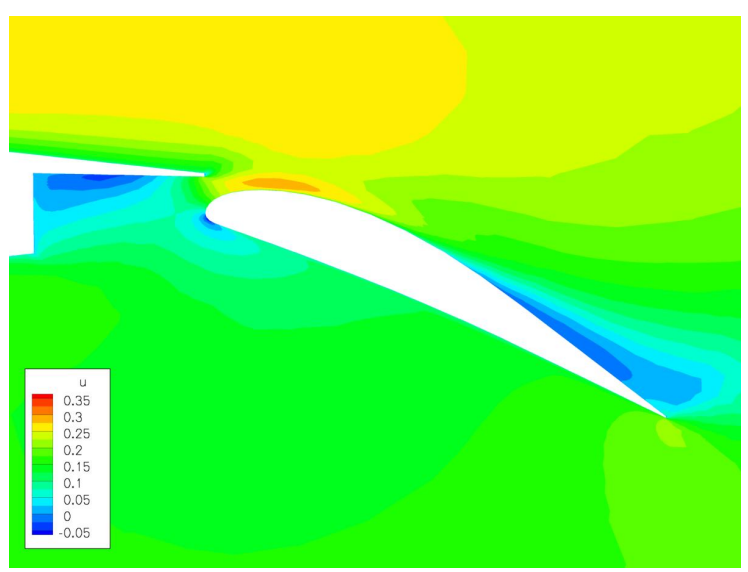

(c) Original coarse grid $u$-velocity.

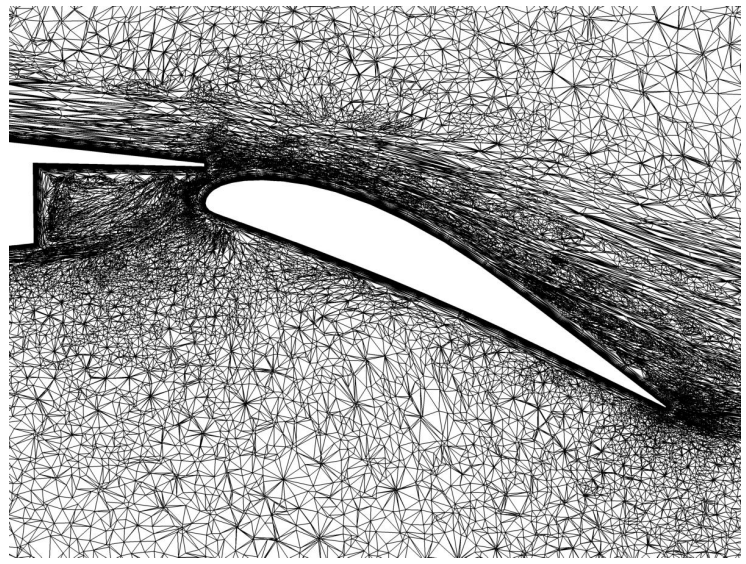

(b) Final adapted coarse grid (11.0M nodes).

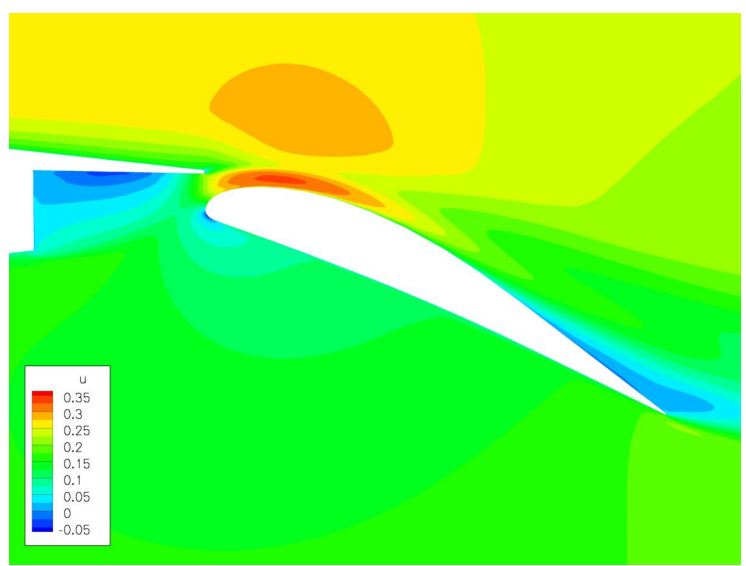

(d) Final adapted coarse grid $u$-velocity.

Figure 20. Slice of volume grid at the $85 \%$ span station for the flap of the original adapted coarse grid, Configuration $1, \alpha=13^{\circ}$. 
adaptation is $23 \mathrm{M}$ nodes.

The simulation adapted with the single grid estimate became unsteady as seen in the iterative convergence plot, Fig. 21(b). The spikes in residual were due to high frequency errors in the interpolated solution of the adapted grid that quickly damped out. The range of coefficient of lift and coefficient of drag are the same for Fig. 21(a) and Fig. 21(b). The lift and drag are oscillating through a significant range of this zoomed-in figure. This case was also run in a time-accurate mode (not shown) but this did not reduce the range of the oscillations. This type of small scale unsteadiness can lead to incorrect sensitivities ${ }^{34}$ because a variable, pseudo-time formulation was used for the flow and adjoint solver, which assumes a steady solution. This problem is exacerbated by the adaptive scheme increasing grid resolution and reducing dissipation induced by insufficient grid resolution. For all cases, the iterative convergence of the SA turbulence model stalled on adapted grids after two adaptations. The source of this stalled iterative convergence is still being investigated. The convergence histories of lift and drag at other angles of attack (not shown) reached steady-state.

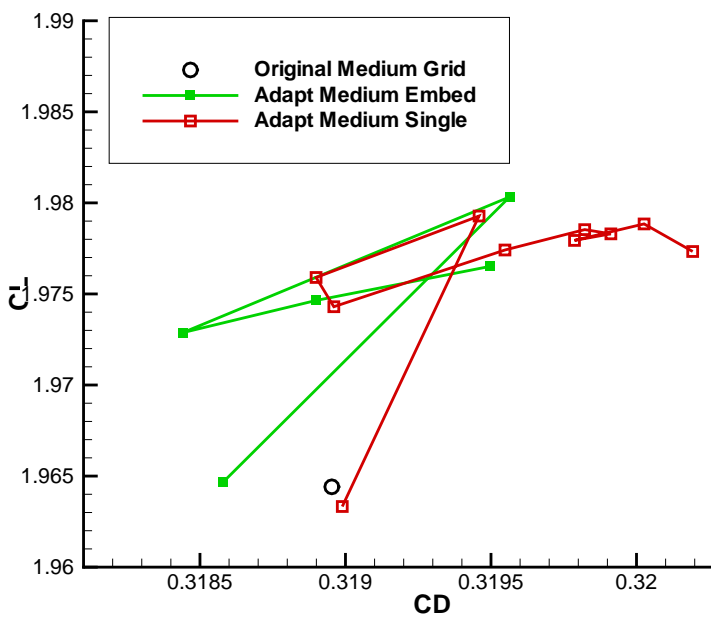

(a) Lift as a function of drag.

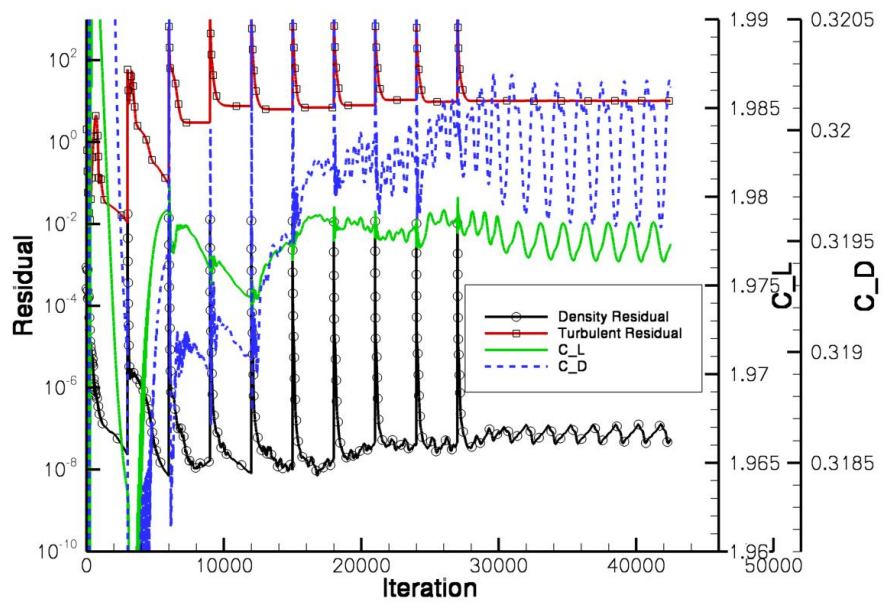

(b) Convergence history of adaptation with single-grid error estimate.

Figure 21. Medium grid adaptation, Configuration 1, $\alpha=13^{\circ}$.

The coefficient of pressure on the flap of the original medium grid and medium grid adapted with the single-grid error estimate are shown in Fig. 22 for the $85 \%$ span station at $\alpha=13^{\circ}$. The adapted grid data is a snap shot of an unsteady simulation, Fig. 21(b). The adapted grid better approximates the experimental coefficient of pressure measurements than the original grid. The indication of separation on the initial medium grid at $x=74.5$ is reduced with adaptation. The trend of reduced separation with grid refinement are also indicated by the grid convergence of the surface pressure coefficients for the Mix(NCTetA) grids, Fig. 12(a). There is less flap separation indicated by the adapted grid than the fine tetrahedral and mixed-element grids, Fig. 14(c).

The surface pressure coefficient is shown in Fig. 23 for the original and adapted grids at $\alpha=28^{\circ}$. This is the same condition as in Fig. 5 and Fig. 13. As seen in Fig. 23(a), the upper surface pressure decreased at the $98 \%$ span station of the main element with grid refinement, showing improved comparison to the experimental measurements and FNS structured grid results, Fig. 5(a). The negative coefficient of pressure peak moved outboard with a slightly more negative value for the adapted grid, Fig. 23(b). The location of the peak was well predicted, but overall levels were less negative than the measurements. This is the opposite of the trend indicated by the unstructured and structured uniform refinement studies, Fig. 13. The difference in trends with grid refinement may be due to the frozen geometry and near wall boundary layer grid of the adaptive results. The small peak near $y=-6$ due to the side of body vortex is better resolved. It is also clearly seen in the structured grid results, Fig. 13(b).

Vorticity magnitude contours at $x$-constant volume slices are shown in Fig. 24 for the original and adapted grids at $\alpha=28^{\circ}$. The structured grids were sliced at the same locations shown in Fig. 6 . These same slices were shown from a different perspective in Fig. 8 for unstructured grids with and without brackets. The vorticity rapidly dissipated downstream for the original medium grid, Fig. 24(a). The vorticity in the 


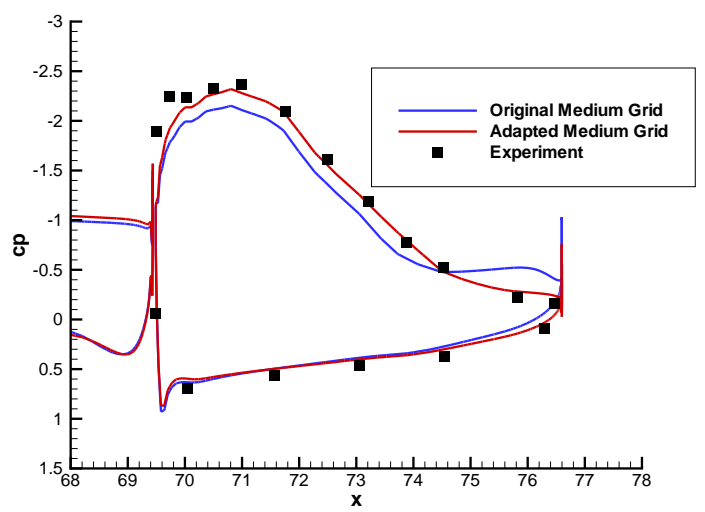

Figure 22. Medium grid flap coefficient of pressure at $85 \%$ span station, Configuration $1, \alpha=13^{\circ}$.

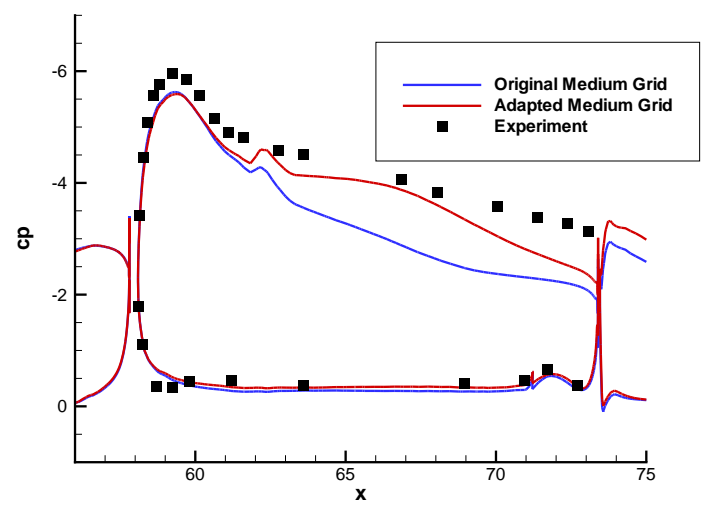

(a) Main element at the $98 \%$ span station

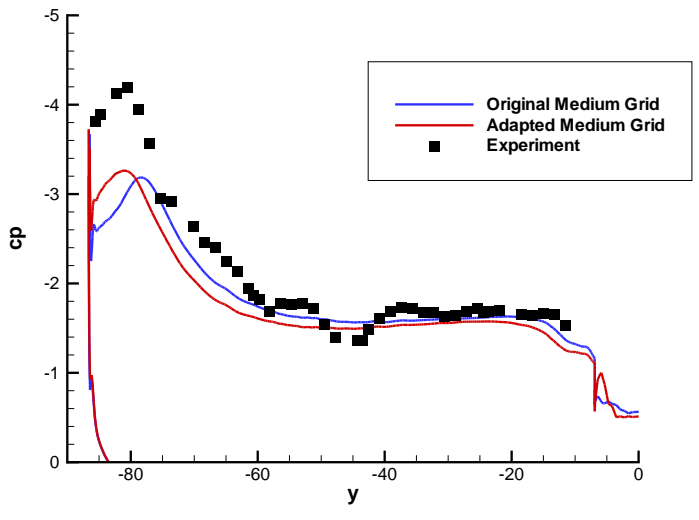

(b) Flap at forward spanwise location

Figure 23. Surface pressure coefficients, Configuration 1, $\alpha=28^{\circ}$, original and adapted grids. 
boundary layer, wake, and vortices was better defined in the adapted grid, Fig. 24(b). The vorticity in the slat wake was better defined for the adapted grid, and the tip and side-of-body vortices were more clearly defined. The original grid slat wake was almost indistinguishable from the main element and flap boundary layers, whereas the adapted grid created improved resolution of the wakes, similar to the C-mesh topology of the structured grid, Fig. 6 .

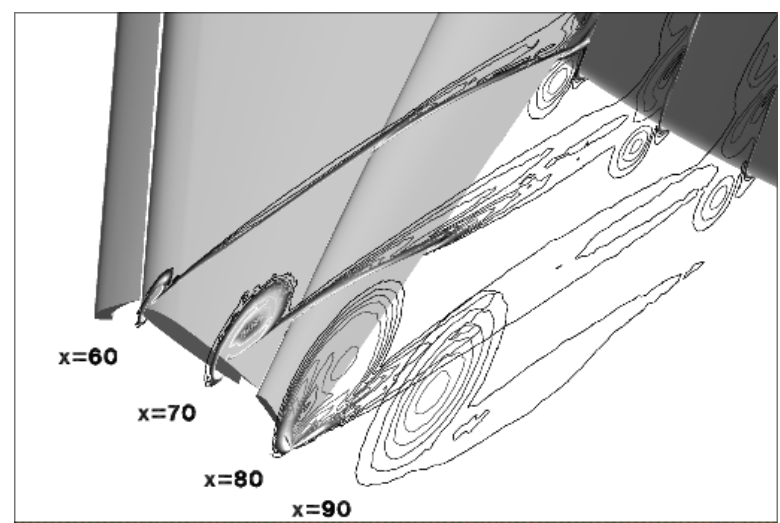

(a) Original medium grid.

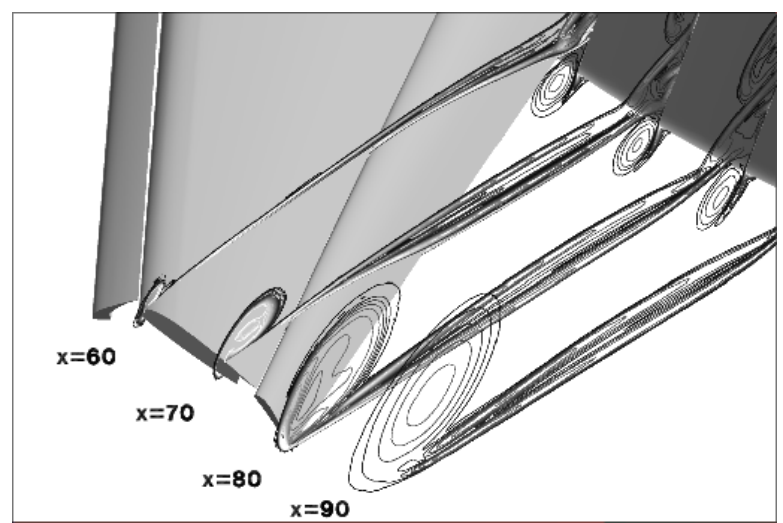

(b) Adapted medium grid.

Figure 24. Vorticity contours showing tip vortex development, FUN3D, Configuration $1, \alpha=28^{\circ}$, SA, medium grid.

This study has highlighted the need for improvements in the current implementation of the output-based adaptation process. The frozen boundary layer limitation of the adaptation mechanics may be preventing the calculation of the fine grid result starting with a coarse initial grid, Fig. 17 (b). The $\alpha=13^{\circ}$ case became unsteady as the grid was refined, Fig. 21(b), which indicates the need for grid adaptation schemes compatible with time-accurate simulation. Monitoring the iterative convergence and adaptation process for the large number of cases required to compute the angle of attack sweep can be improved. The turbulence model failed to converge on the adapted grids, which will require further examination.

\section{Conclusions}

The contributions of two Reynolds-averaged Navier-Stokes codes - one structured and one unstructured to the $1^{\text {st }}$ AIAA CFD High Lift Prediction Workshop were described. Two types of submissions were made: (a) a set of standard runs on coarse, medium, and fine grids, exploring the effects of grid, code, turbulence model, and viscous term treatment; and (b) a set of output-based grid-adapted solutions, exploring the influence of automated grid refinement. These submissions follow the current state-of-the-art for CFD applied to high-lift applications. The latter submissions represent active development at the leading edge of CFD capabilities.

Among the standard runs, there were several different combinations of grid, code, turbulence model, and viscous term treatment. The majority of these were official workshop submissions, while one set of computations on the grids provided by JAXA (including brackets) was only performed for this paper. The SST turbulence model was found to produce more separation and lower lift than the SA model. The latter model's results agreed better with experiment. Two independently-generated structured grids were found to yield different behaviors near $C_{L, \max }$ when using the SST model, although the particular aspects of the grids that might have caused the difference were not discovered.

The effect of grid refinement was assessed for $\alpha=13^{\circ}$ and $28^{\circ}$. Among all the runs that used the SA model, the forces and moments did not approach a converged infinite-grid solution. In other words, the use of different grids and different codes yielded a non-negligible spread in results even as the grids were refined. The spread was greater at the higher angle of attack. A tetrahedral grid exhibited somewhat greater grid sensitivity than a mixed element version of the same grid (for which boundary layer tetrahedra were merged into prisms). The use of the approximation neglecting viscous cross derivative terms yielded lower lift than full Navier-Stokes. A key difference was in the prediction of the wing tip vortex; the thin-layer type of approximation yielded a more diffuse vortex and hence not as much suction force over the wing tip upper surface region. The use of full Navier-Stokes yielded results that agreed much better with experiment. The unstructured code tended to exhibit more trailing edge flap separation than the structured code at $\alpha=13^{\circ}$, 
but the particular unstructured grid used also had an effect on this flow feature. The effect of brackets was explored in a qualitative sense. Including brackets yielded lower lift levels moving the results away from the measurements, but produced some features that bore greater similarity to experiment, such as a spanwise "scalloping" of the pressures over the flap.

The output-adaptive process was driven with both embedded-grid and single-grid error estimates. The single-grid error estimation procedure also included the option of adapting at a constant number of control volumes, which yields an optimized grid for a given computational resource. The near-wall regions of the grids were frozen due to limitations in the grid adaptation mechanics. The embedded-grid error estimate was applied to the entire set of workshop angles of attack with the workshop-provided coarse grid. The singlegrid procedure was applied to the same conditions with the medium workshop grid. Both error estimation procedures were compared at $\alpha=13^{\circ}$ for the medium grid. Grid adaptation showed improved resolution of velocity, surface pressures, and vorticity magnitude. Changes in these quantities indicated a better resolution of tip vortices, side-of-body vortices, gap jets, and wakes for the adapted unstructured grids. The extent of boundary layer separation on the upper surface of the flap is inferred by flow velocity and surface pressures. The trend of reduced separation with adaptive grid refinement compares favorably with the uniform grid refinement studies. Limitations discovered in the current output-adaptive approach were detailed to guide further development of the method.

\section{Acknowledgments}

The single grid error estimation procedure was extended to viscous flows by Mr. Joseph Derlaga, a graduate student trainee in the NASA Langley Computational AeroSciences Branch. The authors would like to thank Dr. Mitsuhiro Murayama of the Japan Aerospace Exploration Agency (JAXA) for providing the JAXA unstructured grids in a format that was compatible with the FUN3D flow solver. The authors wish to thank the NASA Fundamental Aeronautics Program for its continued support.

\section{References}

\footnotetext{
${ }^{1}$ Mavriplis, D. J., Vassberg, J. C., Tinoco, E. N., Mani, M., Brodersen, O. P., Eisfeld, B., Wahls, R. A., Morrison, J. H., Zickuhr, T., Levy, D., and Murayama, M., "Grid Quality and Resolution Issues from the Drag Prediction Workshop Series," AIAA Paper 2008-930, 2008.

${ }^{2}$ Chaffin, M. S. and Pirzadeh, S., "Unstructured Navier-Stokes High-Lift Computations on a Trapezoidal Wing," AIAA Paper 2005-5084, 2005.

${ }^{3}$ Mavriplis, D. J., "Grid Resolution Study of a Drag Prediction Workshop Configuration Using the NSU3D Unstructured Mesh Solver," AIAA Paper 2005-4729, 2005.

${ }^{4}$ Peraire, J., Peirò, J., and Morgan, K., "Adaptive Remeshing for Three-Dimensional Compressible Flow Computations," Journal of Computational Physics, Vol. 103, No. 2, 1992, pp. 269-285.

${ }^{5}$ Baker, T. J., "Mesh Adaptation Strategies for Problems in Fluid Dynamics," Finite Elements in Analysis and Design, Vol. 25, No. 3-4, 1997, pp. 243-273.

${ }^{6}$ Aftosmis, M. J. and Berger, M. J., "Multilevel Error Estimation and Adaptive h-Refinement for Cartesian Meshes with Embedded Boundaries," AIAA Paper 2002-0863, 2002.

${ }^{7}$ Alauzet, F. and Loseille, A., "High-Order Sonic Boom Modeling Based on Adaptive Methods," Journal of Computational Physics, Vol. 229, No. 3, 2010, pp. 561-593.

${ }^{8}$ Rannacher, R., "Adaptive Galerkin Finite Element Methods for Partial Differential Equations," Journal of Computational and Applied Mathematics, Vol. 128, 2001, pp. 205-233.

${ }^{9}$ Pierce, N. A. and Giles, M. B., "Adjoint Recovery of Superconvergent Functionals from PDE Approximations," SIAM Review, Vol. 42, No. 2, 2000, pp. 247-264.

${ }^{10}$ Venditti, D. A., Grid Adaptation for Functional Outputs of Compressible Flow Simulations, Ph.D. thesis, Massachusetts Institute of Technology, 2002.

${ }^{11}$ Fidkowski, K. J. and Darmofal, D. L., "Output Error Estimation and Adaptation in Computational Fluid Dynamics: Overview and Recent Results," AIAA Paper 2009-1303, 2009.

${ }^{12}$ Krist, S. L., Biedron, R. T., and Rumsey, C. L., "CFL3D User's Manual (Version 5.0)," NASA TM 208444, NASA Langley Research Center, June 1998.

${ }^{13}$ Roe, P. L., "Approximate Riemann Solvers, Parameter Vectors, and Difference Schemes," Journal of Computational Physics, Vol. 43, 1981, pp. 357-372.

${ }^{14}$ Spalart, P. R. and Allmaras, S. R., "A One-Equation Turbulence Model for Aerodynamic Flows," La Recherche Aerospatiale, Vol. 1, No. 1, 1994, pp. 5-21.

${ }^{15}$ Mentor, F. R., "Two-Equation Eddy-Viscosity Turbulence Models for Engineering Applications," AIAA Journal, Vol. 32, No. 8, Aug. 1994, pp. 1598-1605.

${ }^{16}$ Anderson, W. K. and Bonhaus, D. L., "An Implicit Upwind Algorithm for Computing Turbulent Flows on Unstructured Grids," Computers and Fluids, Vol. 23, No. 1, 1994, pp. 1-22.
} 
${ }^{17}$ Anderson, W. K., Rausch, R. D., and Bonhaus, D. L., "Implicit/Multigrid Algorithm for Incompressible Turbulent Flows on Unstructured Grids," AIAA Paper 95-1740, 1995.

${ }^{18}$ Nielsen, E. J., Aerodynamic Design Sensitivities on an Unstructured Mesh Using the Navier-Stokes Equations and a Discrete Adjoint Formulation, Ph.D. thesis, Virginia Polytechnic Institute and State University, 1998.

${ }^{19}$ Venkatakrishnan, V., "Convergence to Steady State Solutions of the Euler Equations on Unstructured Grids with Limiters," Journal of Computational Physics, Vol. 118, No. 1, 1995, pp. 120-130.

${ }^{20}$ Diskin, B., Thomas, J. L., Nielsen, E. J., Nishikawa, H., and White, J. A., "Comparison of Node-Centered and CellCentered Unstructured Finite-Volume Discretizations: Viscous Fluxes," AIAA Journal, Vol. 48, No. 7, July 2010, pp. 1326-1338.

${ }^{21}$ Nielsen, E. J., Lu, J., Park, M. A., and Darmofal, D. L., "An Implicit, Exact Dual Adjoint Solution Method for Turbulent Flows on Unstructured Grids," Computers and Fluids, Vol. 33, No. 9, 2004, pp. 1131-1155.

${ }^{22}$ Nielsen, E. J. and Anderson, W. K., "Recent Improvements in Aerodynamic Design Optimization on Unstructured Meshes," AIAA Journal, Vol. 40, No. 6, 2002, pp. 1155-1163.

${ }^{23}$ Park, M. A., Anisotropic Output-Based Adaptation with Tetrahedral Cut Cells for Compressible Flows, Ph.D. thesis, Massachusetts Institute of Technology, Sept. 2008.

${ }^{24}$ Eisenstat, S. C., Elman, H. C., and Schultz, M. H., "Variational Iterative Methods for Nonsymmetric Systems of Linear Equations," SIAM Journal on Numerical Analysis, Vol. 2, April 1983, pp. 345-357.

${ }^{25}$ Park, M. A., "Three-Dimensional Turbulent RANS Adjoint-Based Error Correction," AIAA Paper 2003-3849, 2003.

${ }^{26}$ Lee-Rausch, E. M., Park, M. A., Jones, W. T., Hammond, D. P., and Nielsen, E. J., "Application of a Parallel AdjointBased Error Estimation and Anisotropic Grid Adaptation for Three-Dimensional Aerospace Configurations," AIAA Paper 2005-4842, 2005.

${ }^{27}$ Park, M. A. and Darmofal, D., "Parallel Anisotropic Tetrahedral Adaptation," AIAA Paper 2008-917, 2008.

${ }^{28}$ Andren, J. and Park, M. A., "Output Based Grid Adaptation for Viscous Flow," 18th International Meshing Roundtable Research Notes, Sandia National Lab, Oct. 2009, pp. 1-5.

${ }^{29}$ Park, M. A. and Carlson, J.-R., "Turbulent Output-Based Anisotropic Adaptation," AIAA Paper 2010-168, 2010.

${ }^{30}$ Johnson, P. L., Jones, K. M., and Madson, M. D., "Experimental Investigation of a Simplified 3D High Lift Configuration in Support of CFD Validation," AIAA Paper 2000-4217, 2000.

${ }^{31}$ McGinley, C. B., Jenkins, L. N., Watson, R. D., and Bertelrud, A., "3-D High-Lift Flow-Physics Experiment - Transition Measurements," AIAA Paper 2005-5148, 2005.

${ }^{32}$ Slotnick, J. P., Hannon, J. A., and Chaffin, M., "Overview of the 1st AIAA CFD High Lift Prediction Workshop," 49th AIAA Aerospace Sciences Meeting, American Institute of Aeronautics and Astronautics, Reston, VA (submitted for publication).

${ }^{33}$ Rumsey, C. L., Long, M., Stuever, R. A., , and Wayman, T. R., "Summary of the First AIAA CFD High Lift Prediction Workshop," 49th AIAA Aerospace Sciences Meeting, American Institute of Aeronautics and Astronautics, Reston, VA (submitted for publication).

${ }^{34}$ Krakos, J. A. and Darmofal, D. L., "Effect of Small-Scale Output Unsteadiness on Adjoint-Based Sensitivity," AIAA Journal, Vol. 48, No. 11, Nov. 2010, pp. 2611-2623. 